my journal manuscript No.

(will be inserted by the editor)

\title{
Measurements of Photospheric and Chromospheric Magnetic Fields
}

\author{
Andreas Lagg • Bruce Lites • Jack Harvey • \\ Sanjay Gosain - Rebecca Centeno
}

\begin{abstract}
The Sun is replete with magnetic fields, with sunspots, pores and plage regions being their most prominent representatives on the solar surface. But even far away from these active regions, magnetic fields are ubiquitous. To a large extent, their importance for the thermodynamics in the solar photosphere is determined by the total magnetic flux. Whereas in low-flux quiet Sun regions, magnetic structures are shuffled around by the motion of granules, the high-flux areas like sunspots or pores effectively suppress convection, leading to a temperature decrease of up to $3000 \mathrm{~K}$. The importance of magnetic fields to the conditions in higher atmospheric layers, the chromosphere and corona, is indisputable. Magnetic fields in both active and quiet regions are the main coupling agent between the outer layers of the solar atmosphere, and are therefore not only involved in the structuring of these layers, but also
\end{abstract}

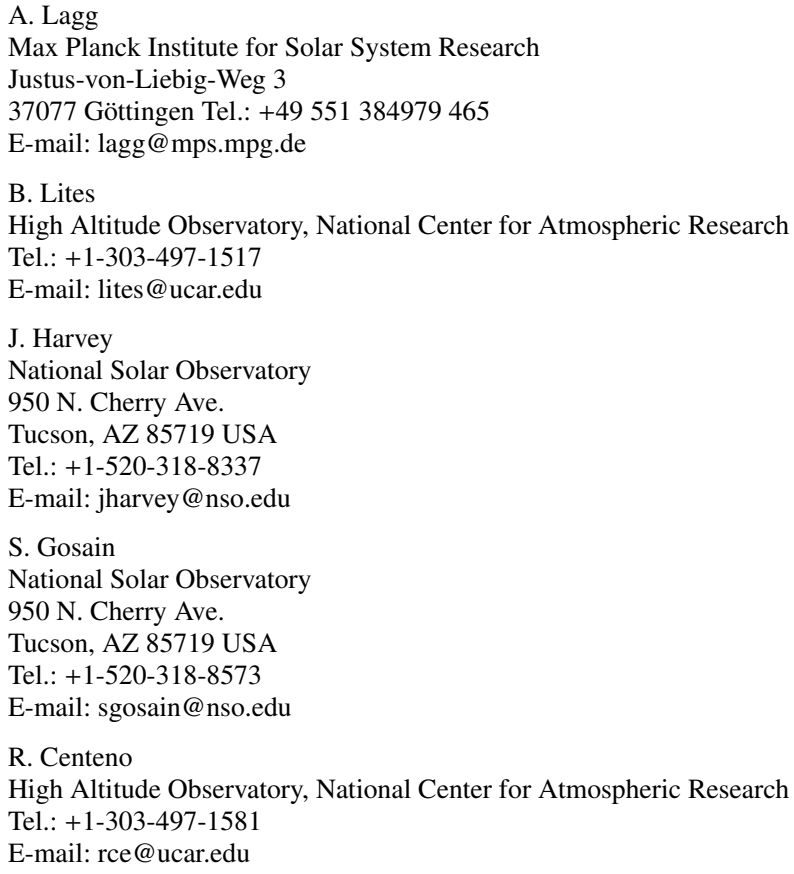


for the transport of energy from the solar surface through the corona to the interplanetary space.

Consequently, inference of magnetic fields in the photosphere, and especially in the chromosphere, is crucial to deepen our understanding not only for solar phenomena such as chromospheric and coronal heating, flares or coronal mass ejections, but also for fundamental physical topics like dynamo theory or atomic physics. In this review, we present an overview of significant advances during the last decades in measurement techniques, analysis methods, and the availability of observatories, together with some selected results. We discuss the problems of determining magnetic fields at smallest spatial scales, connected with increasing demands on polarimetric sensitivity and temporal resolution, and highlight some promising future developments for their solution.

Keywords Sun $\cdot$ magnetic field $\cdot$ chromosphere $\cdot$ photosphere $\cdot$ measurement $\cdot$ observations $\cdot$ spectro-polarimetry

\section{Introduction}

The energy produced by nuclear fusion in the core of our closest star, the Sun, is transported into space almost exclusively by photons emitted from the solar surface. In the absence of solar magnetic fields, this energy flux would likely be nearly constant. It is the solar activity cycle, manifested in the reversal of the solar magnetic field with a 22 -year periodicity, which is the main contributor to the variation of the solar energy flux impinging on earth. This variation is only at the order of less than one percent (Solanki et al.2013), but this is sufficient to affect our natural and technical environment in a significant manner. Spectacular eruptions of high energy particles in so-called coronal mass ejections also have the solar magnetic field as their driver. Any reliable space weather forecast, important for example to avoid damage to Earth orbiting satellites, requires the understanding of the energetics in the magnetized solar atmosphere. A robust determination of the solar magnetic field in all atmospheric layers is therefore mandatory.

The transition from the regime dominated by convective gas and plasma motions to the one driven by magnetic forces takes place between the photosphere and the chromosphere. In this layer, the plasma $\beta$ (i.e., the ratio of gas pressure to magnetic pressure) becomes unity. Extremely fast physical processes on size scales at and well below the resolution limit of current solar telescopes, like reconnection or wave dissipation, occur ubiquitously in active regions but also in places of low solar activity. As a logical consequence, solar instrumentation development aims for the determination of the magnetic field at highest spatial and temporal resolution directly above, in and below the $\beta=1$ layer (Kleint and Gandorfer 2015, this issue).

Since in situ measurements of the field using magnetometers in these deep layers of the solar atmosphere are impossible, we have to rely on remote-sensing observations. The information about the magnetic field must be extracted from the radiation emitted from the solar surface and the layers above it. Instruments in the near ultra-violet, the visible regions (where the energy flux from the photosphere and the chromosphere is highest), and the infrared regions of the solar spectrum record the intensity and polarization profiles of Fraunhofer lines. The conditions in the solar atmosphere encoded in these spectral lines are deciphered by the combination of advanced instrumentation and sophisticated analysis techniques.

For more than a century the Zeeman effect has been used to reliably determine the magnetic field in the photosphere (Hale 1908, Stenflo 2015, this issue). Enhanced instrumental 
capabilities allowed one to extend the measurements from regions with strong Zeeman signals, like sunspots or pores (Rempel and Scharmer 2015 this issue), down to small-scale events and also towards higher atmospheric layers: the chromosphere and the corona (Trujillo Bueno and Landi degl'Innocenti 2015, Wiegelmann et al. 2015, this issue). Especially in these weak field environments, the Hanle effect has gained outstanding importance during the last two decades. In some special spectral lines the advantages of both effects are combined, and allow for continuous measurements from weak, unresolved magnetic fields in the sub-gauss regime up to strong, kilo-gauss field in sunspots.

The unambiguous interpretation of observations using these two effects requires the exact treatment of the physical mechanisms behind them. The radiative transfer equation must be solved, the population of the energy sub-levels in the relevant atoms of the solar atmosphere must be computed, and the effect of radiation and collisions must be considered (de la Cruz Rodriguez et al. 2015, this issue). In addition, the tiny imprints of weak, small scale magnetic fields in the polarization signals of spectral lines require advanced instrumentation with cutting-edge technology in, e.g., optical engineering and detector design.

\section{Overview of Solar Magnetic Field Measurement}

Several methods have been used to estimate the properties of the magnetic field in various regions of the solar atmosphere. The assumption that density structures and their dynamics are entrained along or guided by the magnetic field has long been used to infer magnetic properties from observations of the solar corona, prominences and chromospheric fibrils (e.g. Bigelow 1889). Frequencies of oscillation of features thought to be tied to magnetic fields have been used to estimate magnetic field strengths (e.g. Hyder 1966, Ballester 2014). Various properties of solar radio emission are used to deduce magnetic field characteristics in the chromosphere and corona (see review by Kundu 1990). In addition to these remote sensing observations, direct measurement of heliospheric magnetic fields has been done for decades, and by 2024 we can expect in situ magnetic field measurements as close as 8.5 solar radii above the solar surface (Solar Probe Plus 2015).

By far the majority of solar magnetic field remote sensing measurements have been made by observations of the Zeeman and Hanle polarization effects on atomic spectral lines. Some reviews of solar spectropolarimetry methods include Stenflo (1994, 2013) and Keller (2002). The vastly larger general field of polarimetry measurements and techniques is frequently reviewed. Some recent reviews of special note include Trippe (2014), Snik et al. (2014), and Rodenhuis et al. (2014).

It is useful to consider the key elements of solar spectropolarimetry as a sequence from the source to the inferred measurement: source magnetic field $\rightarrow$ radiative transfer $\rightarrow$ atmosphere $\rightarrow$ telescope $\rightarrow$ calibration $\rightarrow$ imaging optics $\rightarrow$ polarization modulator $\rightarrow$ wavelength selector $\rightarrow$ detector $\rightarrow$ data recording $\rightarrow$ calculation of source polarization $\rightarrow$ inference of source magnetic field properties. Our task is to determine the source magnetic field as functions of 3D spatial position and time from observations of the Stokes vector elements that convey intensity and polarization information as functions of $2 \mathrm{D}$ spatial position, spectral wavelength, and time. This is a big challenge. In the future, it may be possible to derive more robust $3 \mathrm{D}$ information by using simultaneous stereoscopic observations from space. One could discuss each of the key elements of solar spectropolarimetry in detail but space constraints prevent that. Here we give a simple overview with focused attention on some topics of current interest. 


\subsection{Contemporary Observational and Research Areas}

At present, one may identify four major domains of solar polarimetry research driven by technical tradeoff considerations and scientific interests.

1. High spatial resolution, with the goal of exploring small-scale magnetoconvection.

2. High polarimetric sensitivity, for studies of weak magnetic fields including the chromosphere and corona.

3. Full solar disk synoptic sequences, for investigations of large-scale magnetic field evolution, the solar dynamo and solar cycle, and for space weather applications.

4. Fast cadence, to better understand solar activity such as flares, CMEs, spicules, filaments, etc.

There is not enough light available to meet all these needs simultaneously using available instrumentation or methods. Tradeoffs are required, and to guide these tradeoffs various system considerations are useful. A spectropolarimetric solar observation can be represented as an 5D hypercube with time $(t)$ as one dimension and Stokes vector elements, which represent polarization information $(I, Q, U, V)$ as one additional dimension. The remaining three dimensions are two plane-of-the-sky coordinates $(x, y)$ and wavelength $(\lambda)$. Usually this datacube is drawn at one instant of time with edges $x, y, \lambda$ and cube volume elements containing one of the four Stokes vector elements. Ideally we would like to completely fill such a datacube with a single, brief snapshot in order to obtain observations with the most efficient use of available light. Hagen and Kudenov (2013) have very usefully reviewed progress toward snapshot spectral imaging technology and techniques. In practice, current measurements of solar magnetic fields are mostly made using spatially-scanning slit spectrometers or wavelength-scanning narrow band filters. In the former case observing time speed is compromised in order to maintain spectrum line profile integrity at the expense of non-simultaneous spatial sampling. In the latter case observing speed is emphasized but the profile integrity is compromised by the need to observe spectra at different wavelengths and polarizations at different times. The history of magnetic field estimation is full of attempts to mitigate these tradeoffs and their compromises.

\subsection{Time Constraints}

The duration of an observation is important for two main reasons. First, the Stokes vector must be measured accurately at each datacube element before any observation conditions change. Second, the acquisition of a spectropolarimetric data cube must be made sufficiently fast compared to the evolutionary time scale of the magnetic structure observed. These requirements conflict with both the acquisition of a sufficient number of photons for low-noise Stokes polarimetry and construction of the spatial/spectral images quickly enough to suppress noise from solar feature evolution and ground-based seeing or space-based pointing jitter. Thus, progress in solar magnetic feature measurement is strongly influenced by such tradeoffs, but it is also strongly linked to technology improvements that enhance the efficiency of observations. In current practice, intensity measurements in at least four different polarization modulation states are needed to fully define a Stokes vector measurement. A polarization modulator device sequentially produces the different states. Measurement noise arises irreducibly not only from variations in the number of photons detected (shot-noise), but also spuriously from any image intensity or geometry changes during the modulation sequence. A goal is to not add significant spurious noise in excess of the shot noise. For 
ground-based observations, atmospheric seeing is a major source of noise. Measurements of the frequency spectrum of seeing effects on solar images (Rimmele 2000) show large power at low frequencies but also that significant noise is present at frequencies in excess of $100 \mathrm{~Hz}$.

Practical experience shows that polarization noise produced by atmospheric seeing may be reduced by rapid acquisition of modulated solar images. Spatial resolution and site conditions are important factors, but completing a polarization modulation sequence in $10 \mathrm{~ms}$ (from the ground) or a few seconds (from space - depending on the frequency spectrum of pointing jitter) is highly desired for minimizing spurious noise. Ground-based slit spectrograph polarimeters using fast polarization modulation can reach noise levels (uncertainty of $Q, U$, or $V$ relative to $I$ ) of order $10^{-5}$ while ground-based filter polarimeters are usually limited to $10^{-3}$ due to an additional time penalty of acquiring many images at different wavelengths and polarization states during which observation conditions change. Adaptive optics and reconstruction techniques for ground-based filter images can reduce the spurious noise caused by seeing. For both ground and space filter observations the evolving pattern of solar intensity and polarization produce polarization noise that depends on the properties of the instrument and the acquisition sequence of the various wavelength and polarization state images.

\subsection{Solar Polarimetry is Light-Starved}

To determine the basic structure of strong magnetic features like sunspots, noise levels of $10^{-2}$ of the continuum intensity are acceptable. Some high sensitivity work requires noise of the order $10^{-5}$, or even smaller. Considering just shot noise, $10^{4}$ to $10^{10}$ photons must be collected to reach these noise levels. Suppose we want to make a photospheric observation at disk center using two spatial pixels per diffraction limit with an overall instrument efficiency of $10 \%$. The temporal modulation typically used for polarization measurements requires us to accept a displacement of no more than 0.05 pixel on the imaging device during the observation, otherwise spurious noise is introduced to the data. Under these conditions and assuming a typical photospheric velocity of $5 \mathrm{~km} \mathrm{~s}^{-1}$, Fig. 1 1 a shows the maximum exposure time allowed at three different wavelengths and a range of telescope apertures. At the small aperture size limit shown (GONG) exposure times of about 10 seconds can be used, whilst at the large size limit (DKIST), 60-160 ms are the longest exposures allowed.

If we use two spectral samples per spectral resolution element of $\lambda / \Delta \lambda=90000$ and assume an overall system transmission of $10 \%$, then Fig. 1p shows the maximum signalto-noise ratio $(\mathrm{S} / \mathrm{N})$ for Stokes I that can be obtained in the continuum computed using the maximum available exposure times. Note that the $\mathrm{S} / \mathrm{N}(I / \Delta I$ or $I / \Delta p)$ will be lower for $Q, U, V(\Delta p \approx \sqrt{3} / \Delta I)$ and in the darker parts of spectral lines. It is obvious that achieving high polarimetric sensitivity in diffraction limited observations is very difficult.

\subsection{Strategies to Improve Efficiency}

The grim outlook shown in Fig. 1p has motivated many tradeoffs to be able to secure useful high-resolution, high-precision measurements of solar magnetic fields. Several of these were used in the first solar magnetographs. One may, for example, use larger spatial and spectral samples, combine signals from more than one spectrum line, use sparse sampling of the spectrum, slice the incoming solar image into a format better suited to the spectrometer, 

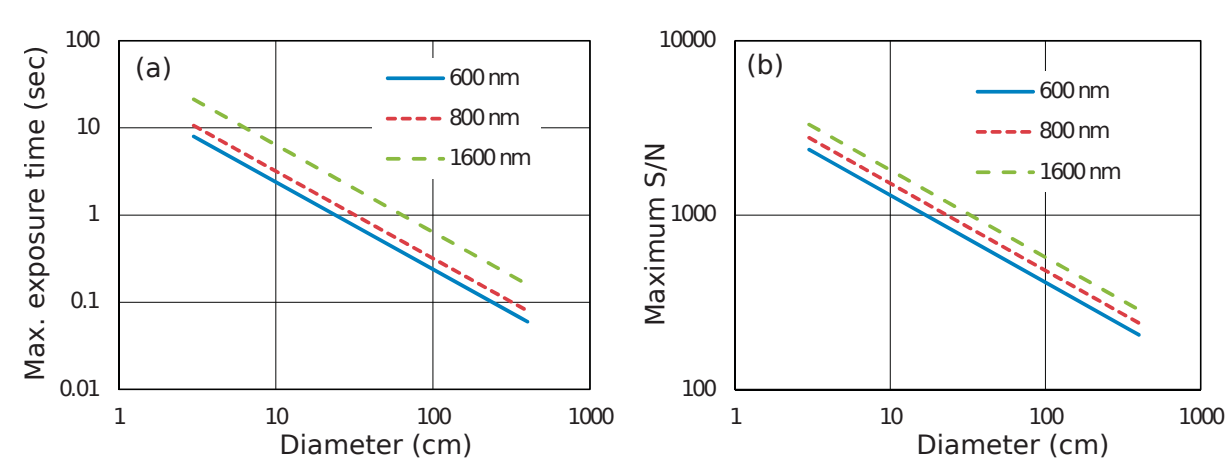

Fig. 1 Left (a): Maximum exposure time for diffraction-limited disk-center photospheric observation constrained by $<0.05$ pixel motion of target at $5 \mathrm{~km} \mathrm{~s}^{-1}$. Right (b): Maximum signal to noise ratio per exposure for diffraction-limited photospheric observations at disk center resulting from the number of photons per pixel impinging the detector within the maximum exposure time.

and/or select a wavelength compatible with high overall instrumental efficiency. Dual-beam polarimetry was introduced so that all the photons are used all the time. As detector technology advanced, better quantum efficiency and higher read-out speeds were achieved all the time (in addition to greatly reducing crosstalk among polarization states arising from residual image motion and blurring).

There are only a few chromospheric and coronal spectral lines that are useful for magnetography, but many lines forming in the photosphere are available. The photospheric photon flux per spectrum line Doppler width peaks at near-IR wavelengths around $1 \mu \mathrm{m}$ so this should be an optimum wavelength for observations. However, IR spectrum lines tend to be weak compared to those at shorter wavelengths (though this is counteracted by stronger Zeeman splitting at long wavelengths), and spatial resolution for a given telescope aperture is smaller at longer wavelengths. Historically, lines in the visible part of the spectrum have been favored mainly owing to the availability of lower-cost detectors with high quantum efficiency. At present, the optimum wavelength for photospheric magnetic field Zeeman effect measurements ranges from the yellow to the near infrared.

One long-used strategy is to observe only circular polarization (Stokes $V$ ), from which the line-of-sight component of the magnetic field can be estimated. Provided the magnetic elements are spatially resolved, to first order the strength of the circular polarization signal depends linearly on the source magnetic field strength, while the linear polarization signal (Stokes $Q$ and $U$ ) has a quadratic dependence, and for modest field strengths it is rather weak.

The availability of detectors with large numbers of pixels that can be read out fairly quickly has stimulated clever techniques to fill the image/polarization/wavelength hypercube in single snapshots (Hagen and Kudenov 2013). The basic idea is to divide the large space on the detector in ways that allow recording spectral, spatial, and polarization information simultaneously. One older example first used photographically (Martin et al. 1974) is as follows. Instead of using a small format detector in a spectrograph having one long entrance slit, a large format detector allows one to use many entrance slits each producing a spectrum of a different part of the solar image. Fig. 2 is an early example of what such an observation would look like. Overlapping of the output spectra is mitigated by using a blocking filter with a narrow pass band. So instead of scanning a solar image with one slit, using $\mathrm{N}$ slits reduces the required scan time to $1 / \mathrm{N}$, a significant efficiency improvement. There are dozens of 


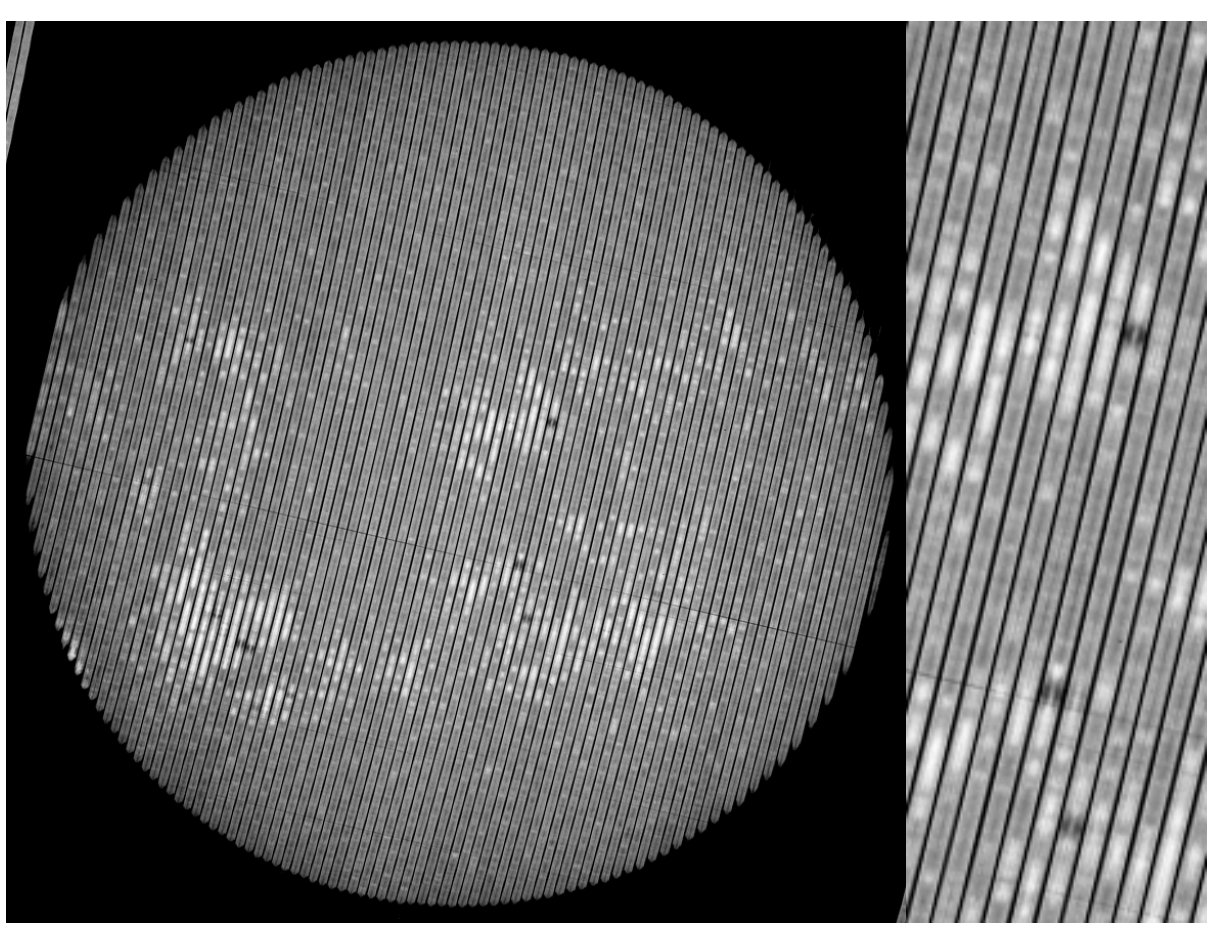

Fig. 2 An early example of trading sparse spatial sampling to gain spectral coverage. Spectroheliogram and zoom showing individual K-line spectra at numerous positions of the entrance slit (Meudon, 1938 August 8).

other clever ideas to improve efficiency based on large format detectors. Many of the ideas use fiber optics, microlens, micropolarizer, and microfilter arrays and other modern optical technologies. It is almost inevitable that a new generation of solar magnetographs will utilize some of these efficiency improvements.

Detector performance is key to magnetic field measurement. Fortunately, detector technology continues to improve rapidly. High efficiency sometimes requires conflicting properties. These include: high quantum efficiency over a large range of wavelengths, large pixel count, large dynamic range, excellent linearity, fast readout, no image smear, excellent modulation transfer function, no image lag (signal retention between successive readouts), no etalon fringing, and, if possible, pixel level on-chip demodulation capability. Manufacturers have devised various architectures based on converting photons to charges in a detection layer followed by conversion of the charges to a measurable voltage. In some devices the detection layer is stacked above a layer of readout electronics. In others all the components are essentially in one layer. In some devices (e.g. CCD, most CMOS) the readout process destroys the original charge while in others (e.g. CID, DEPFET, ZIMPOL, sCMOS) the charge is shuffled and not necessarily destroyed immediately by the readout process. To obtain high speed, the sensor area is divided into smaller regions and readout is accomplished simultaneously through multiple channels. 


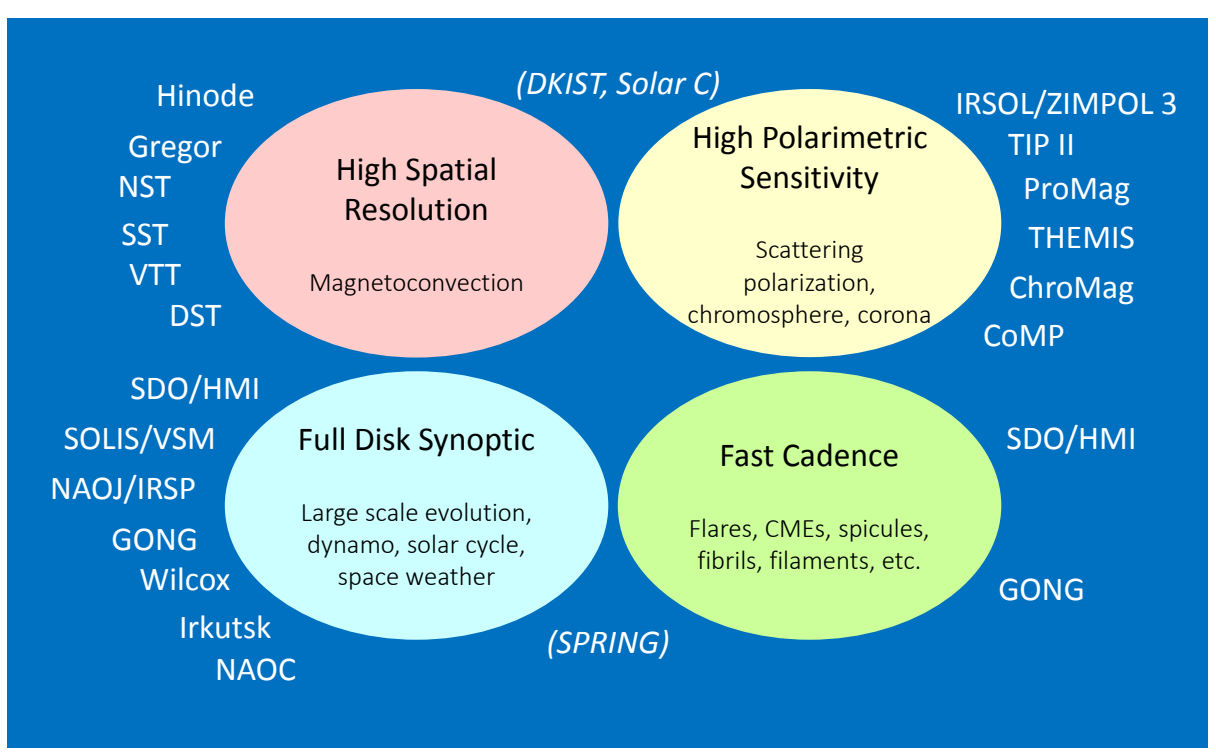

Fig. 3 Domains of current solar spectropolarimetry and a sampling of telescope/instrument names clustered to the domain for which they are best suited.

\subsection{State of the Art}

Fig. 3 summarizes the major domains of solar spectropolarimetry, and some telescopes/instruments in use (or planned in parentheses) placed close to the domains for which they were designed. This is not a full listing (for example, a few years ago there were more than 20 instruments capable of measuring the chromospheric magnetic field). The figure is intended to suggest the large range of capabilities available for measuring the solar magnetic field limited primarily by financial and personnel resources and the advance of technology.

\section{Ground-Based Techniques}

Ground based observations have several advantages over space- or balloon-borne observatories. Their large aperture allows for highest spatial resolution, they can be equipped with complex instrumentation with a diverse selection of observing modes in a multitude of spectral lines, elaborate calibration schemes can be applied, and virtually no limits on data rates do exist. The only impediments to observing from ground in past few decades were the atmospheric seeing effects and the diurnal cycle. The latter has been very successfully mitigated by establishing globally distributed observing networks with identical instrumentation such as GONG, which can be run for extremely long time periods by constant maintenance and upgrades. On the other hand the techniques that are used to overcome seeing effects has seen tremendous progress. In the following we will give examples of the current state-ofthe-art in high resolution imaging techniques, narrow band two-dimensional spectrometers, and high precision polarimeters. 


\subsection{Longitudinal Magnetometry: Still a Viable Tool}

The term magnetometry has been loosely used in solar physics for the measurement of magnetic fields in the solar atmosphere. However, in reality we can only remotely sense the solar magnetic field by analyzing the photons emitted from the solar atmosphere and applying diagnostic techniques such as Zeeman and Hanle effect to the analyzed photons. Depending on the extent of analysis of the collected photons one can infer either complete vector or just longitudinal component of the magnetic field. The strength of longitudinal magnetometry is its simplicity in interpreting the measured signal, resulting from the almost linear dependence of the longitudinal Zeeman effect to the line-of-sight component of the magnetic field (Stenflo 2015, this issue). Complex, model dependent techniques, like inversions, can often be avoided, and still the fine structure of the solar atmosphere can be retrieved (Stenflo 1994). Babcock (1953) used the polarization properties of the Zeeman components of the spectral line to measure the projection of the magnetic field vector along the line-of-sight. The instruments that use this method to map the longitudinal magnetic field of the Sun are popularly known as Babcock type magnetographs. Longitudinal magnetographs have been used extensively for studying the distribution and evolution of magnetic flux on the Sun at a variety of spatial and temporal scales. Modern longitudinal magnetographs based on two dimensional image sensors exist in various flavors (e.g., SOLIS, Hinode SP/NFI, GONG, $\mathrm{MDI}$ ), and are still very popular for variety of studies such as quiet sun magnetism, flux emergence, active region evolution, magnetic helicity flux estimation, flare related changes, polar field strengths, coronal field extrapolations and so forth.

\subsection{The Age of Vector Magnetic Field Measurements}

In contrast to longitudinal measurements the transverse fields are more challenging to measure and, even more, to interpret. The Zeeman effect induced linear polarization signal is proportional to the square of the transverse field strength and is therefore weaker compared to the circular polarization signal which is proportional to the longitudinal field strength. This different response of the measured signals to the orientation of the magnetic field results often in ambiguous interpretations. For example, the distribution of the magnetic field orientation in weak field regions is still under debate as to whether the weaker fields are predominantly horizontal or vertical (Borrero and Kobel 2013). In active regions, especially near the polarity inversion line, the transverse component of the field becomes strong, often accompanied with a highly non-potential or sheared magnetic field topology. The presence of free or excess magnetic energy above the potential field energy is a necessary condition for the occurrence of energetic phenomena such as flares and coronal mass ejections (CMEs). It is therefore necessary to measure the full magnetic field vector in solar active regions in order to estimate the non-potentiality of the magnetic field and its evolution leading to flare and or CME. Further, it is now well known that solar active regions show a weak hemispheric bias in their magnetic field twist (Pevtsov et al. 1994). The twist of the magnetic field can be derived by using various proxies such as force-free parameter $\alpha$, current helicity, magnetic helicity, chirality of filament barbs, sense of rotation of chromospheric superpenumbral whirls around sunspots.

Most of the above mentioned proxies require the measurement of the full magnetic vector field. The origin of the helicity in active regions and their hemispheric bias is still an open question. Various mechanisms have been proposed to explain it, for example its origin in the solar dynamo itself, or during the rise of buoyant flux tubes via their interaction with 


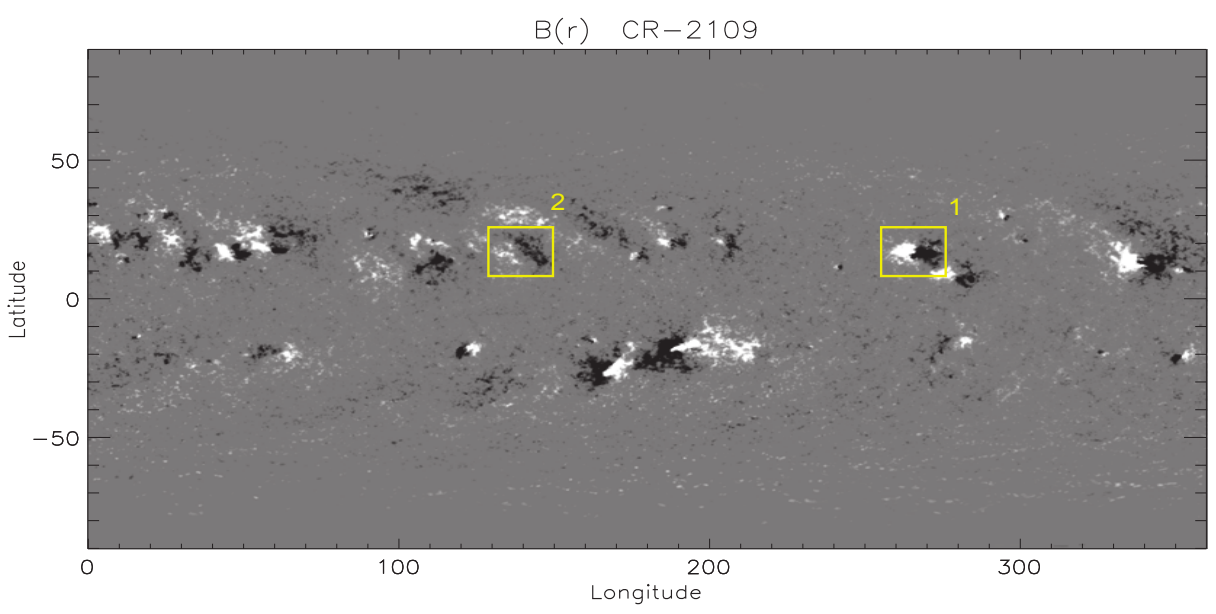

Fig. 4 Synoptic Carrington map of the vector magnetic field component $\mathrm{B}_{r}$ synthesized using full-disk SOLIS/VSM vector magnetograms is shown for CR-2109 (the $\mathrm{B}_{\theta}$ and $\mathrm{B}_{\phi}$ components are not shown). The map is scaled between $\pm 100 \mathrm{G}$ with positive (white) values of $\mathrm{B}_{r}$ pointing upward. The yellow square boxes ' 1 ' and ' 2 ' show examples of a typical compact active region and a diffuse decaying region, respectively.

turbulent convection, or after the flux emergence due to reconnection near the polarity inversion line. Some authors think that the solar dynamo itself is responsible for the generation of helicity, others believe that the turbulent convection interacting with the buoyant flux tubes as they rise towards the surface is the culprit, and a third group thinks that helicity is generated via reconnection processes when the emerged flux interacts with the ambient magnetic properties of the solar corona.

A systematic monitoring of vector fields on the solar disk is therefore needed to understand the relation between magnetic twist and other observables, as well as to build up statistics. Synoptic observations of the magnetic field vector over the full disk derived from full Stokes polarimetry in the photospheric Fe I $630.1 \mathrm{~nm}$ line are routinely done by the SOLIS/VSM instrument. Fig. 4 shows the synoptic Carrington map of the photospheric field (radial component $\mathrm{B}_{r}$ ) generated using the daily fulldisk measurements by SOLIS/VSM (Gosain et al. 2013). Such maps are useful, for example, for monitoring the hemispheric helicity trends (Gosain et al. 2013), their relation with the kinetic helicity of subsurface flows derived from helioseismology (Komm et al.|2014, Komm and Gosain 2015), global nonlinear force-free field (NLFFF) extrapolations (Tadesse et al. 2014b a) and so on. In addition to synoptic vector field measurements of the full disk it is also desirable to have highly sensitive magnetic field measurements in certain areas of the solar disk such as polar regions and beneath non-active region filaments that also can erupt and be associated with a CME.

\subsubsection{Filter-Based or Imaging Instruments}

As mentioned in Sec. 2 the magnetic field is determined from multi-dimensional spectropolarimetric observations: for every pixel of a 2-dimensional map the spectrum and the temporal evolution should be recorded, resulting in a 4-dimensional data cube for each of the four Stokes components $(I, Q, U, V)$. Since detectors can only record two-dimensional images, a choice of the order of the sampling must be made. Two different approaches are commonly taken, the filter-based and spectrograph-based measurement. Both are eventually 
leading to same output but are fundamentally different in that the imaging instruments sample two spatial dimensions simultaneously and spectral samples are obtained sequentially, while in the case of spectrograph-based instruments one spatial dimension (along the slit) and the spectral dimension are obtained simultaneously and the second spatial dimension is obtained sequentially by scanning the slit laterally. The fourth dimension of the data cube, time, is achieved by the repetition of these measurements.

Examples of current filter type instruments are the Imaging Vector Magnetograph (IVM, Mickey et al.1996), the Interferometric BIdimensional Spectrometer (IBIS, Cavallini 2006), the Solar Vector Magnetograph (SVM, Gosain et al. 2006), the CRisp Imaging SpectroPolarimeter (CRISP) at the SST (Scharmer et al.2008), and the GREGOR Fabry-Pérot Instrument (GFPI, Denker et al. 2010). Tunable imaging spectrometers come in various flavors. For example, HMI uses a Michelson Interferometer as a filter, and CRISP, IBIS, GFPI, IVM, and SVM use tunable air-gap Fabry-Pérot etalons in single or tandem configuration. The rapid tunability of piezo-mounted air-gap etalons and the high throughput are main advantages of these devices. Further, Fabry-Pérot etalons have the advantage that, unlike for example birefringent filters, they do not contain linear polarizers. This makes dual beam polarimetry possible (see Sec. 3.3 by placing the polarizing beam splitter close to the focal plane of the cameras. Alternatively, one can also choose solid electro-optic crystal based etalons such as lithium niobate $\left(\mathrm{LiNbO}_{3}\right)$ crystal wafer based etalons (Mathew et al. 1998, Debi Prasad and Gosain 2002; Martínez Pillet et al. 2011) where the highly polished thin wafer is coated with highly reflective coatings. Here, the wavelength tuning is based on the change of the refractive index and the thickness of the $\mathrm{LiNbO}_{3}$ crystals by applying different voltages. The advantage of these solid etalons compared to normal, air-spaced etalons is their high refractive index $(\approx 2.28)$, allowing for smaller etalon diameters for a given fieldof-view. For instance, the largest air-gap etalons ( $15 \mathrm{~cm}$ aperture) ever made and used in the Improved Solar Observing Optical Network (ISOON, Neidig et al. 1998) could be replaced by $6.5 \mathrm{~cm}$ aperture $\mathrm{LiNbO}_{3}$ etalons of equivalent throughput. On the other hand their limitations are that they are fragile (specially with larger apertures), have higher absorption losses, and are susceptible to damage if tuned faster than 1000 Volts/s.

Under the assumption that the acquisition of a spectropolarimetric data-cube is faster than the evolution timescale of the solar structures and that of the atmospheric seeing, filterbased instruments provide a snapshot of the magnetic field over the field-of-view. Thus, such measurements are useful for studies of the temporal evolution of magnetic fields at high cadence, ideal for studies about, e.g., flares or waves. Until the beginning of the new millennium, the magnetic field inference from these observations had some limitations in terms of spectral resolution, sparse wavelength sampling, or spectral purity (Lites et al. 1994). However, significant progress has been made since then in improving the performance of these instruments in various aspects, for example by combining two or more etalons in tandem configurations to increase the spectral resolution. Instruments like CRISP, IBIS, TESOS and more recently GFPI, therefore now allow to obtain depth dependent, high spatial resolution vector magnetograms. As an example, Fig. 5 shows the longitudinal field obtained from SST/CRISP observations with twice the angular resolution of Hinode/SOT (reproduced from Scharmer et al. 2013). Such observations are possible by combining several techniques, like adaptive optics, post-facto image reconstruction algorithms and the high spectral resolution and throughput of the CRISP instrument. Due to short acquisition time of the spectropolarimetric dataset from such instruments it is possible to acquire high quality observations even during brief moments of good seeing. 


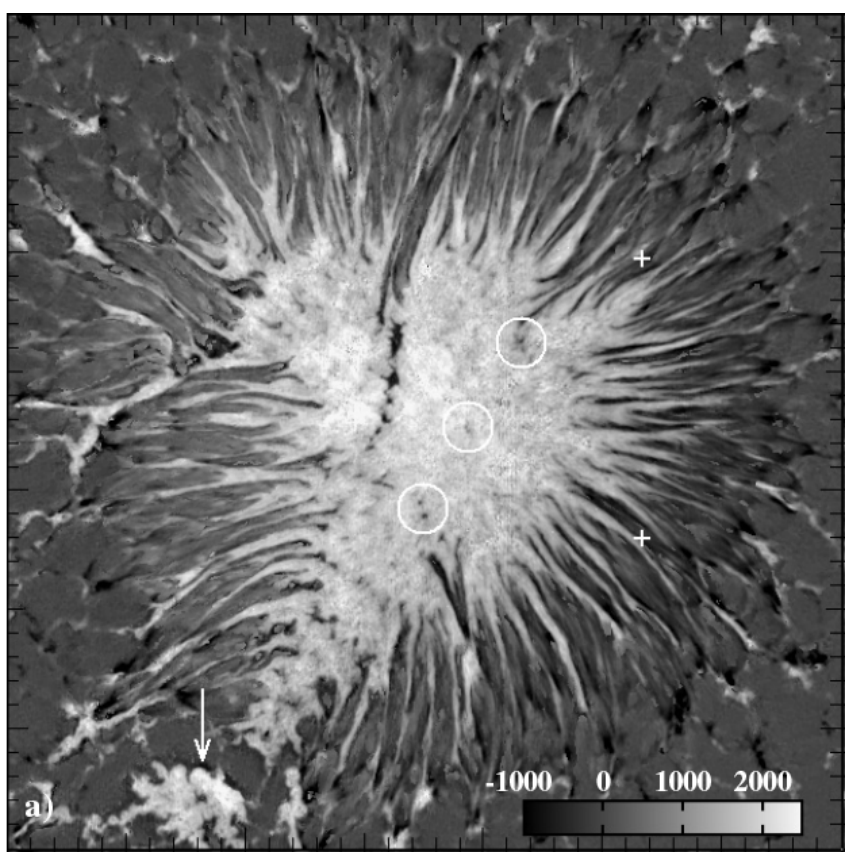

Fig. 5 Longitudinal magnetic field for a $35^{\prime \prime} \times 35^{\prime \prime}$ large FoV at $\tau_{c}=0.95$ derived from NICOLE inversions show the presence of opposite polarity fields in most parts of the penumbra. Such opposite polarity fields are absent in higher layers (e.g., at $\left.\tau_{c}=0.09,0.01\right)$. The long white arrow points towards the disk center. Figure reproduced from Scharmer et al. (2013), Fig. 4(a).

\subsubsection{Stokes Spectrographic Observations}

The spectrograph based instruments sample the spectral dimension simultaneously. Spatial scanning by moving the slit over the field-of-view is needed to build two-dimensional maps. Prominent examples of spectrograph based imaging polarimeters are ASP (Lites 1996), SOLIS/VSM (Keller and Solis Team 2001), SPINOR (Socas-Navarro et al. 2006), TIP (Collados et al. 2007), GRIS (Collados et al.2013). In order to expedite the scanning time, a few variants have been developed, such as the multi-slit spectrograph, that uses multiple slits separated far enough so that the usable range of spectra can be recorded without overlap (e.g., FIRS, Jaeggli et al.|2010). Here, an order-sorting pre-filter must be used to isolate the wavelength range of interest. Other variants can be grouped into two classes: (1) integral field spectrographs (IFS), where the 2D field-of-view is converted to a one dimensional arrangement feeding a regular slit spectrograph, using either an image slicer (Bowen 1938) or a bundle of optical fibres (e.g. DLNIRSP, Elmore et al. 2014), and (2) microlens arrays producing multiple point images out of an extended field-of-view, again feeding a spectrograph with the multiple point sources, oriented such that the dispersion avoids overlapping of the individual spectra (microlens fed spectrograph, M. van Noort, private communication).

Simultaneous wavelength information together with typically higher spectral resolution, higher signal-to-noise ratios and finer wavelength sampling are the main benefits of these instruments. This is of particular advantage when inferring the accurate field strength and orientation in the pixel, including the height stratification of the physical parameters in the line forming region of the solar atmosphere. Sit-and-stare observing modes cater well to the studies of dynamic phenomena, ideally complemented with high temporal resolution slit-jaw imaging. With an IFS the dynamical studies can be extended to a small but 2-dimensional field-of-view. The disadvantage of spectrograph based instruments is that they are not well 
suited for observations of a large field-of-view such as a full AR on time-scales of minutes, which is required by some studies such as flare research.

The ideal solution, therefore, would be to take hybrid approach where the high-quality reflective slit-jaw projection of the spectrograph is used to feed a filter based spectropolarimeter. This allows for information over whole field-of-view with high cadence and limited spectral resolution while the smaller field-of-view sampled by slit (or an IFS) has more detailed spectral information. Real-time processing of the imaging data can help to rapidly re-position the slit to an interesting region, e.g., a flaring kernel or an emerging flux region within the slit-jaw field-of-view.

\subsection{Overcoming Seeing}

One of the biggest challenges for any ground-based measurements is the atmospheric seeing, which changes rapidly within a few tens of milliseconds. The atmospheric seeing can be one of the major sources of spurious polarization signals in sequential measurements (Lites 1987; Leka and Rangarajan 2001). Dual beam measurements, where the orthogonal polarization states are separated by means of a polarizing beam splitter and recorded simultaneously, are essential for reducing the seeing effects in measurements. Unlike single beam setup, the dual beam setup fully utilizes 100 percent of the photons available for polarimetry. However, different optical paths for the measurement of the two polarization states in a dual beam setup might introduce a systematic differential gain/aberrations error, this puts strict constraints on the optical quality of the two optical paths in such a system. Alternatively, if single beam setup is preferred, a very fast modulation scheme with a matching fast camera readout must be used, in order to allow a complete modulation cycle before the seeing changes. However, polarization measurements require accumulation of various modulation cycles to build up the desired $\mathrm{S} / \mathrm{N}$. This usually results in a loss of spatial resolution, since frames taken under variable seeing conditions must be combined to acquire the desired $\mathrm{S} / \mathrm{N}$ ratio. In order to preserve or restore the spatial resolution in such observations we then resort to the high angular resolution techniques as described below.

\subsubsection{Image Correction in Real Time}

The optical systems which can compensate for distorting effects of atmospheric seeing in real-time are known as adaptive optics (AO) systems. Such systems typically have a wavefront sensor (WFS), which provides information on the wavefront distortions. This information is used to control in real-time a corrector optics, typically a combination of tip-tilt mirror and a deformable mirror (Rimmele and Marino 2011; Scharmer et al. 2000). The advantage of such systems is that it (i) enhances the angular resolution in the images, and (ii) allows us to make longer exposures to attain high $\mathrm{S} / \mathrm{N}$ ratio in polarimetric measurements. The latter benefit relieves the requirement for extremely fast camera readouts in order to freeze the atmospheric seeing. However, due to the highly variable nature of seeing and due to time constraints in AO systems, only a limited number of higher order modes can be corrected. Thus, the residual seeing effects still remain, introducing seeing-induced cross-talk (Krishnappa and Feller 2012). Higher modulation frequencies help to reduce the seeing-induced crosstalk (Judge et al. 2004, Gandorfer et al. 2004, Ramelli et al. 2010, Casini et al. 2012, Krishnappa and Feller 2012).

The seeing is generally expressed with the Fried parameter, $r_{0}$, which is a measure of the spatial scale over which the wavefront can be considered approximately distortion-free (rms 
distortion of less than 1 radian). The performance of AO systems depends heavily upon the basic seeing conditions $\left(r_{0}\right)$, the performance being better when $r_{0}$ is larger. Another property of $\mathrm{AO}$ systems is that the quality of real-time corrections is good near the center of isoplanatic patch (typically $\approx 1-2^{\prime \prime}$ ) in the FoV, the patch which is used for the wave front sensing. A correction over a field-of-view much larger then the isoplanatic patch can be achieved with so called multi-conjugate adaptive optics (MCAO) systems (Rimmele et al. 2010). First prototypes of MCAO systems are currently being developed (e.g., Schmidt et al. 2014).

Observations in infrared wavelengths, such as the Fe $1.56 \mu \mathrm{m}$ lines, are best suited for magnetometry, since the magnetic sensitivity scales linearly with the wavelength. A further benefit of observing in this wavelength region is that the atmospheric seeing varies with wavelength such that $r_{0}$ is proportional to $\lambda^{6 / 5}$, i.e., the isoplanatic size is much larger at longer wavelengths.

\subsubsection{Image Reconstruction: Post Processing Techniques}

To further improve the quality of AO corrected data, offline image reconstruction methods, like speckle imaging (von der Luehe 1993), phase diversity (Lofdahl and Scharmer 1994), and multi-object multi-frame blind deconvolution (MOMFBD, van Noort et al. 2005) are applied. These techniques are not limited by isoplantism and can be used to improve image quality over a large field-of-view. Typically they require separate broadband channel images, recorded at a similar wavelength and strictly simultaneously with the narrowband channel images. The high $\mathrm{S} / \mathrm{N}$ ratios in these broadband channel images deliver the additional information required for the reconstruction of the narrowband channel images.

Such reconstruction techniques are essential when narrow band imaging polarimetry is performed as the seeing introduces spatial to spectral crosstalk, and as a consequence spurious polarization signals. Reconstruction methods achieve a good alignment between the pixels of sequentially taken, narrowband images (van Noort et al. 2005), which helps avoid spurious polarization signals. The reconstruction techniques are, however, prone to errors or lead to spurious features when the noise in the data is not properly accounted for, and therefore require accurate calibration of parameters. For further details on speckle polarimetry reader is referred to Keller and von der Luehe (1992) and references therein, and for MOMFBD techniques we refer to van Noort et al. (2005) and Löfdahl and Scharmer (1994).

\subsection{Ground-Based Observing Networks}

A network of identical instrumentation distributed geographically around the globe in order to achieve continuous observations of the Sun has been a very successful concept. The longterm operations of networks, such as Global Oscillations Network Group (GONG, Harvey et al. 1996) or the Birmingham Solar Observing Network (BiSON, Chaplin et al. 1996), have led to a continuous data series of uniform quality. The benefit of running such networks are (i) redundancy of sites (e.g., if one site fails there is still flow of data from other nodes), (ii) a possibility of cross calibration and merging of data, (iii) easy upgradeability and maintenance of instruments. Earlier networks were designed for helioseismology studies, which require long, uninterrupted time series of solar oscillation measurements to achieve high frequency resolution. Nowadays, emphasis is put on long term global solar magnetic field measurements for solar cycle studies and continuous monitoring of vector magnetic fields 
in solar active regions for space weather prediction research. A proposal to set up a new network of identical instruments designed for measuring fulldisk multi-height velocity and magnetic field vector measurements along with high resolution context imaging in different wavelength bands, such as white light, G-band, H-alpha and Calcium K, is currently funded by European Research Council (ERC). This new network is called Solar Physics Research Integrated Network Group (SPRING) (Hill et al. 2013).

\subsection{Examples of the State of the Art and Current Status}

The magnetic field observations got a big boost in the last decade owing to the space based Hinode/SP and SDO/HMI instruments. The current next-big-thing in solar magnetic field measurements is expected from the 4-meter aperture Daniel K. Inouye Solar Telescope (DKIST, Rimmele and ATST Team 2008, Tritschler et al. 2015), currently being built on Maui/Hawaii. Equipped with adaptive optics and very sensitive infrared detectors, DKIST will provide magnetic field measurements with unprecedented sensitivity and spatial resolution. However, since the number of photons per diffraction limit resolution elements remains independent of the telescope aperture size, the telescope will be very sensitive when used as a so-called "photon bucket", i.e., operated at a resolution coarser than its diffraction limit. Another series of large aperture solar telescopes have become fully operational in the last decade: the $1.6 \mathrm{~m}$ New Solar Telescope (NST, Goode et al. 2010) at Big Bear, the $1.5 \mathrm{~m}$ GREGOR telescope on Tenerife (Schmidt et al. 2012), and the $1 \mathrm{~m}$ SST on La Palma (Scharmer et al.2003).

All of these telescopes are equipped with fully operational AO systems, while the development of MCAO systems is being carried out in parallel. In the near future, these MCAO systems will allow for magnetic field measurements at a higher spatial resolution over a much larger field-of-view than conventional AO systems deliver (Rimmele et al. 2010). Also, recent demonstration of ground-layer solar AO by Ren et al. (2015) looks promising.

Specialized detectors designed for high speed polarimetry are capable of reaching a polarization sensitivity of the order of $1 \times 10^{-5}$ of continuum intensity. Examples of such detectors are as below:

- Charge shuffling cameras, e.g., the Zurich Imaging POLarimeter (ZIMPOL), contain hidden buffers for caching the charges on the chip thus allowing to buildup sufficient signal before the actual readout is performed (Povel et al. 1994). Further, since the same pixel is exposed for different modulation states, no differential gain variations are present. The latest version of the ZIMPOL system employs sensors with improved electronics and high sensitivity. New designs/versions of ZIMPOL based on CMOS detectors are also being planned (Ramelli et al.|2010).

- The Fast Solar Polarimeter (FSP, Feller et al. 2014) uses pn-CCD cameras with extremely low readout noise while delivering high frame rates. A polarization sensitivity of below $1 \times 10^{-4}$ of the continuum intensity has already been achieved. The development of these cameras for larger formats (1 megapixel) is a current project at the Max Planck Institute for Solar System Research. 
4 Space-based Techniques

\subsection{Motivation for Measurement of Photospheric Magnetic Fields from Space}

Owing to the circumstance that many aspects of photospheric magnetism have remained largely unresolved, the study of solar magnetism has resulted in an ongoing quest for observations of higher angular resolution. A fundamental scale of the solar atmosphere is the density scale height ( $h_{\rho}$, of order $150 \mathrm{~km}$ in the photosphere). The scale height is the characteristic length for many of the most important hydrodynamic and radiative processes. Resolving photospheric magnetic fields on this scale has been a goal of solar physics for several decades, and the cleanest way to arrive to a spatial resolution at $h_{\rho}$ would be observations from a space-based platform. Unfortunately, the cost of deploying a telescope of the necessary size (diameter of order $1 \mathrm{~m}$ ) in space has been prohibitive to realization of this goal. Parallel efforts from ground-based observatories - larger telescopes, adaptive optics, advanced image restoration techniques - are pushing observations closer to this longsought goal. As a result, the quest for the highest angular resolution from space might now be relegated to a secondary status relative to the benefits of uninterrupted observations with uniformly very high angular resolution. Provided that continuity of observations is achieved, many extremely important science problems of solar physics may be addressed effectively without completely resolving the magnetic fields with resolution equivalent to $h_{\rho}$.

Another motivation for space-based observations is access to the hemisphere of the Sun not visible from Earth (or near-Earth orbit), including detailed study of magnetic fields at the solar poles. Imaging instrumentation has been flown (e.g., the STEREO mission), but so far no observations of photospheric magnetic fields have been carried out from such a vantage point, although one such mission is in development (see Sec. 4.5.)

A less obvious motivation for space observations of solar magnetism is support of coordinated observing campaigns. Science objectives that require diverse observations from multiple observatories (both space- and ground-based) have a much higher chance of success because the space-based components of the campaign have a predictably high probability of success.

\subsection{Challenges of Space-Based Magnetic Field Measurements}

The development of any space mission is a costly and lengthy process, often necessitating international collaboration in order to make the program possible within budgets of the individual participating countries. International collaborations increase the complexity of management of a space instrumentation program, but they also broaden the pool of available scientific and engineering talent. Many of the missions providing measures of photospheric magnetic fields (as summarized below) result from significant international collaboration.

The technical challenges of space instrumentation are significant in comparison to groundbased observing. Weight and power are always at a premium, as is physical size. Space instrumentation must be resilient under extremes of temperature and radiation exposure never encountered on the ground. The vacuum environment can lead to contamination of optical surfaces, so careful attention must be paid to the outgassing properties of materials for space flight. Furthermore, complex instrumentation must be ultra-reliable and multiply redundant to reduce the possibility of a single-point failure that would bring an end to the mission. These challenges of space instrumentation drive the high development cost and result in significant compromises in design. As a rule, the simpler the design and the fewer 
the moving parts, the more reliable the instrument becomes. As a result of simplicity, space instrumentation usually has far less flexibility of operation than comparable ground-based instrumentation.

To date, space instrumentation for measurement of photospheric magnetism has carried out measurements of the Zeeman effect in the visible spectrum only. There is little motivation for observing photospheric fields in ultraviolet lines because the ratio of magnetic splitting to the Doppler width decreases linearly with decreasing wavelength. Furthermore, several other factors render observations at ultraviolet wavelengths more difficult, from both scientific and technical standpoints. Conversely, the advantages of observing in the infrared are countered by the cost of deploying proportionately larger telescopes in order to achieve spatial resolution equivalent to that of an instrument operating in the visible.

Solar observations require high angular resolution, so the measurement of magnetic fields over the spatial extent of even a single active region demands very high data rates. Full Stokes polarimetry at multiple wavelengths is necessary for measurement of the vector magnetic field, hence, at least four times the data volume of intensity-only measurements are required. All of this data must be transmitted to the ground at a cadence appropriate for monitoring the evolution of the target solar features. In order to confront the data requirements in the face of limited telemetry, the polarization data are usually compressed on-board. The compression leads to some loss of information that would not be present in equivalent ground-based measurements. Even with compression, limitations imposed by telemetry remain a limitation to the quantity of data that may be acquired during any given time period.

\subsection{Past and Ongoing Space Missions}

To date there have been only three successful space missions capable of measurements of the photospheric magnetic field. Two of those, $\mathrm{SoHO}$ and $S D O$, carried synoptic instruments with full-disk capability intended for precision Doppler velocity measurements for helioseismology in addition to sensing the magnetic field. The third mission, Hinode, has instrumentation optimized for high-resolution study of magnetic fields as a primary objective. These three missions are described in more detail below, and an example of one significant scientific accomplishment is provided for each.

\subsubsection{Solar and Heliospheric Observatory/Michelson Doppler Imager (SoHO/MDI)}

The SoHO/MDI instrument (Scherrer et al. 1995) was primarily intended for helioseismology, but fortunately the capability for measurement of circular polarization, and hence the line-of-sight component of the magnetic apparent flux density $B^{L}$ app, was retained in spite of pressure to "de-scope" the instrument owing to budgetary considerations. SoHO/MDI operated from December 1995 to April 2011 and therefore provided coverage of the magnetic field spanning more than one complete solar cycle.

The optical layout of SoHO/MDI is shown in Fig. 6 The heart of the instrument, the Michelson interferometers, had heritage from the Fourier Tachometer (Brown 1981), a groundbased instrument for helioseismology. The rotatable waveplates between the interferometers allowed the device to tune over the $0.5 \AA$ bandpass of the Lyot filter centered on the Ni I line at $6768 \AA$. The full-width half-maximum (FWHM) of the combined system was listed at $94 \mathrm{~m} \AA$, and in typical operation SoHO/MDI sampled the line at five wavelengths separated by $75 \mathrm{~m} \AA$. 


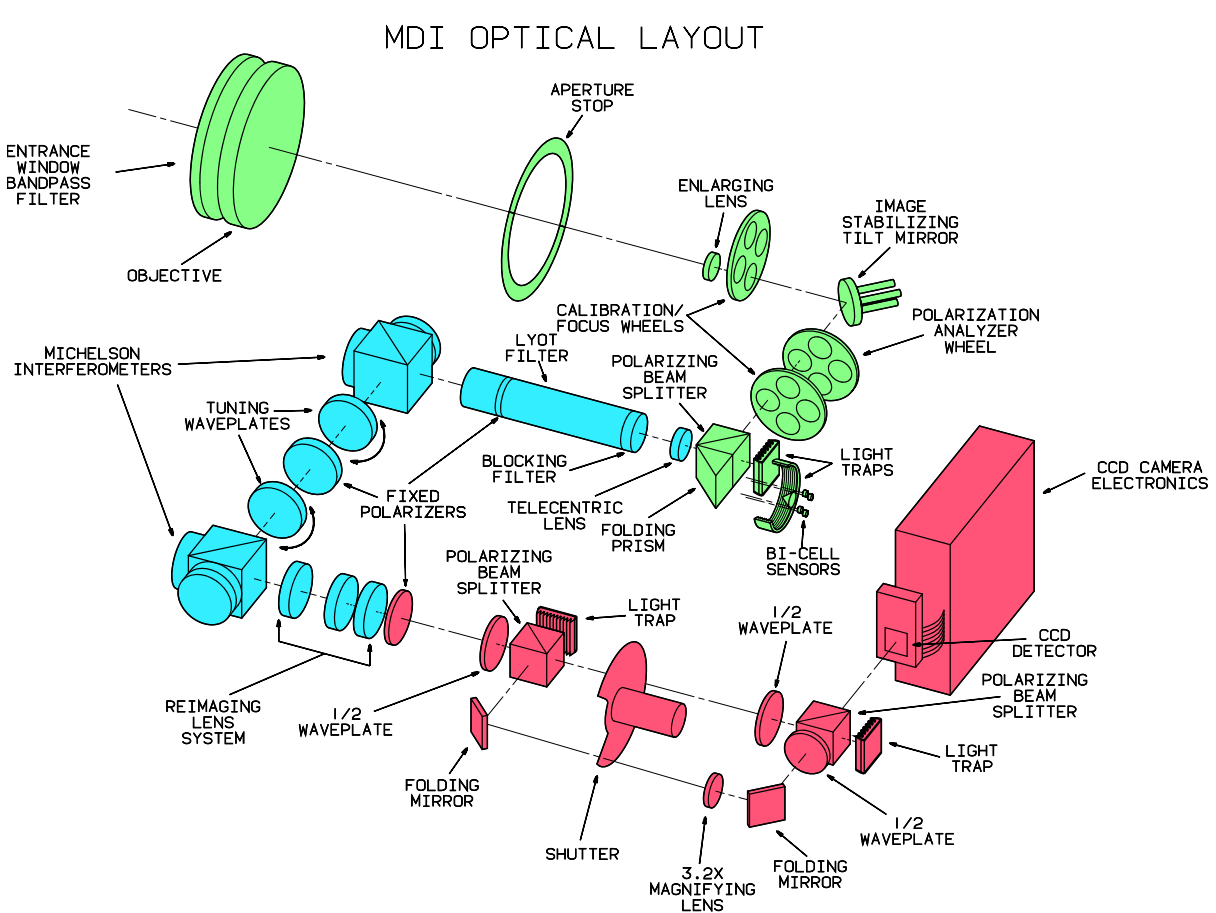

Fig. 6 The optical layout of the SoHO/MDI instrument is shown. The wavelength isolation was accomplished by a pair of Michelson interferometers preceded by a Lyot filter. Circular polarization analysis was accomplished by alternately rotating quarter-wave retarders into the beam with the polarization analyzer wheel. Diagram taken from Scherrer et al. (1995).

The emphasis on helioseismology led to several aspects of this instrument that were not optimal for precision measures of photospheric magnetic fields: performing the polarization analysis in by alternately inserting separate quarter-wave plates, the use of a spectrum line with less than optimal magnetic sensitivity, a spectral resolution a factor of 2-3 times greater than the typical Doppler width of the Ni I line in the photosphere, and limited angular resolution ( 4 arcsec in full-disk mode, $1.2^{\prime \prime}$ in high resolution mode). That being said, owing to its station at the first Earth-Sun Lagrangian point L1, SoHO/MDI was the first instrument to provide an uninterrupted sequence of longitudinal magnetograms of the Sun at a regular cadence and uniform quality. This instrument facilitated the study of the evolution of active regions in a way never before realized.

The magnetogram data from $\mathrm{SoHO} / \mathrm{MDI}$ were (and are still) being used extensively to understand solar phenomena, as witnessed by the more than 800 abstracts citing the MDI instrument between 1996 and 2015. The numerous applications of the data from this instrument facilitated such diverse studies as extrapolation of photospheric fields to compare with structures in the chromosphere and corona, studies of active region evolution including measures of injection of helicity into the upper solar atmosphere, polar fields, flare-induced magnetic field changes, the decay of active regions, and many more.

The science made possible uniquely by this space experiment is illustrated by the statistical study of the orientation axis of bipolar regions (Stenflo and Kosovichev 2012). They examined over 73000 active regions from 1995-2011. This selection included regions with bipolar strength (or flux) ranging from just above the typical flux of ephemeral active re- 
gions to the largest observed regions. They found that the distribution of tilt angles (Joy's law) with solar latitude obeys the same relationship independently of the strength of the dipole (or net flux) of the region. This behavior led the authors to conclude that the tilt of active regions is established at the source of the dynamo giving rise to the active regions, not in the buoyant rise through the convection zone as was previously hypothesized. Furthermore, the authors showed examples (representing just a few percent of the total number of active regions) that did not obey the Hale hemispheric polarity law. The authors then conclude that these exceptions "...rule out the possibility of well-defined, coherent toroidal flux systems as a source of all active regions, even the large ones".

\subsubsection{Solar Dynamics Observatory/Helioseismic and Magnetic Imager (SDO/HMI)}

The Solar Dynamics Observatory (SDO), launched in February 2011, carries as one of its complement of instruments the Helioseismic and Magnetic Imager (HMI, Scherrer et al. 2012, Schou et al. 2012). This instrument is so similar in design to the $\mathrm{SoHO/MDI}$ that no optical layout of the system is provided herein, but it incorporates a number of features that represent significant upgrades from $\mathrm{SoHO} / \mathrm{MDI}$ :

- A different spectral line is observed: Fe r $6173 \AA$. Not only is this line isolated within a fairly clean region of the spectrum to enable precision helioseismology, but it is also a normal Zeeman triplet with high magnetic sensitivity.

- Polarization analysis is carried out with rotating retarders mounted permanently in the beam. This arrangement allows for a higher precision polarimetry than SoHO/MDI. Additionally, these waveplates allow for measurement of the full Stokes vector, so the instrument is capable of vector magnetometry.

- The angular resolution of the instrument is $1^{\prime \prime}\left(0.5^{\prime \prime}\right.$ pixels $)$, with the field-of-view covering entire solar disk.

- Two CCD cameras are used, one is dedicated to helioseismology, the other is dedicated to polarimetry. The data system operates at a higher cadence than that of $\mathrm{SoHO} / \mathrm{MDI}$.

As the name of the instrument implies, this instrument has a much greater scientific emphasis on magnetometry than the SoHO/MDI did. Besides continuing the helioseismic function beyond the end of the $\mathrm{SoHO} / \mathrm{MDI}$ program, $\mathrm{SDO} / \mathrm{HMI}$ brings a new dimension in continuous vector magnetometry of the full disk. This instrument makes it possible, for the first time, to perform synoptic studies of the vector magnetic field of every active region on the disk. The work of Liu et al. (2014) serves as one example of a study involving the SDO/HMI vector magnetogram data of many active regions. In that work they sought to validate, with data of higher resolution and uniform quality, the rather weak hemispheric preference for sign of the twist of active region fields as reported by several authors who used ground-based data. As a measure of twist they adopted a $B_{z}{ }^{2}$-weighted average of the force-free parameter $\alpha$ (obeying $\nabla \times \mathbf{B}=\alpha \mathbf{B}$ ) over the active region. Fig. 7] shows the result of their analysis of 151 active regions appearing during the first 3.5 years of the current activity cycle. This study demonstrates a very clear hemispheric preference for sign of the magnetic helicity. The high cadence of $S D O / \mathrm{HMI}$ vector magnetic field measurements allowed them to select for study observations of active regions as they traversed the solar central meridian, thereby avoiding any bias that could arise as a result of viewing angle with respect to solar longitude.

Being a very similar cousin to the $S O H O / M D I$ instrument, the $S D O / H M I$ instrument is optimized for helioseismology rather than precision polarimetry. As a result, it has some limitations for measurement of the vector magnetic field. Firstly, the instrument has a rather low duty cycle and therefore it does not use the available photons efficiently. The signal-to-noise 


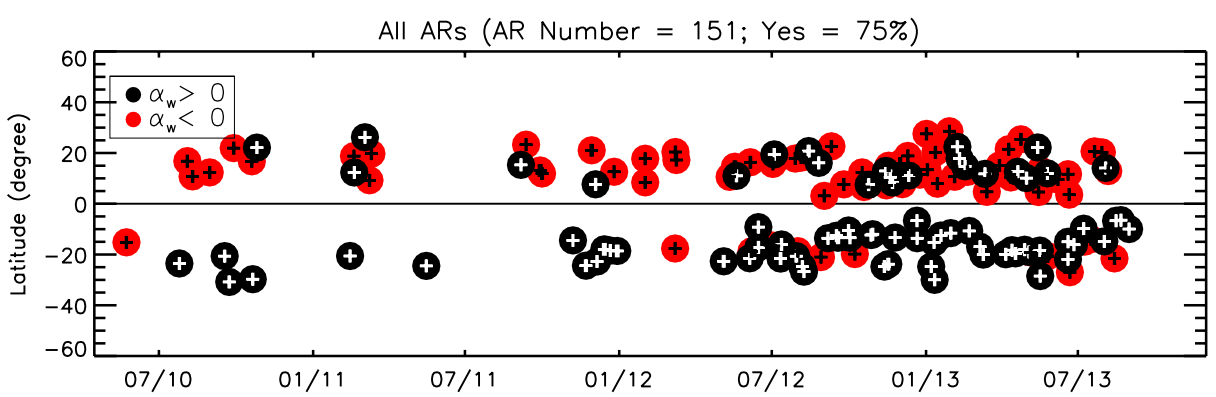

Fig. 7 The latitudinal distribution of the sign of the $B_{z}{ }^{2}$-weighted average $\alpha_{w}$ of the force-free parameter $\alpha$ is indicated as a function of solar latitude and time during the rise phase of Solar Cycle 24. The figure results from analysis of vector magnetograms obtained with $\mathrm{SoHO} / \mathrm{MDI}$. Sign of this proxy of magnetic helicity is indicated by color. For this distribution of 151 active regions, 75\% show the hemispheric preference for helicity suggested by previous studies of ground-based data. Diagram adapted from Liu et al. (2014).

ratio of typical polarimetric observations is such that studies of the vector magnetic field in quiet regions are extremely difficult at best. Second, the instrument is a single-beam polarimeter with a slow polarization modulation cycle. This means that there will be crosstalk among the Stokes parameters arising from uncompensated image motion and evolution of the solar scene. Third, only one spectral line is sampled, and that with six samples of the line profile. Each wavelength sample is subject to a filter bandpass of $76 \mathrm{~m} \AA$. Instruments optimized for Stokes polarimetry sample the complete spectral profile of two or more lines having different sensitivities to the Zeeman effect, and do so with a spectral resolution comparable to the Doppler width of the line in question (typically 30-40 m $\AA$ for photospheric lines). The compromises of the $S D O / \mathrm{HMI}$ spectral sampling and resolution result in greatly reduced sensitivity to subtle features of the Stokes profiles that would otherwise assist in precision measures of the magnetic field vector. They also prevent the accurate extraction of magnetic filling factors in the data reduction process, so the standard SDO/HMI data inversions assume unit filling factor. For regions outside of sunspots, the extracted field strength is then actually a measure of the average field strength over the resolution element - a measure that is lower than the actual field for the usual case where intense magnetic elements are not resolved spatially. Furthermore, the inferred field inclination will be more transverse to the line-of-sight than the actual field. Thus one must anticipate significant systematic errors in the inferred magnetic field vector for regions outside of sunspots. These errors may or may not be significant depending upon the nature of the science question being addressed. For studies that are highly sensitive to such errors, one should use data from instruments specifically optimized for Stokes polarimetry, such as the Solar Optical Telescope on the Hinode mission described in the following. Furthermore, limited spectral sampling hinders the extraction of vertical gradients of physical parameters that might otherwise be available to analysis in finely-sampled Stokes profiles, and the limited wavelength range of these samples precludes measurement of strongly Doppler-shifted fields or very strong Zeeman splitting in sunspot umbrae.

\subsubsection{Hinode SOT/FG and SOT/SP}

The Hinode mission (Kosugi et al. 2007) is the first space mission carrying instrumentation specifically optimized for precision Stokes polarimetry. For this purpose it carries the $50 \mathrm{~cm}$ Solar Optical Telescope (SOT: Tsuneta et al. 2008) with capability of both spectrographic 


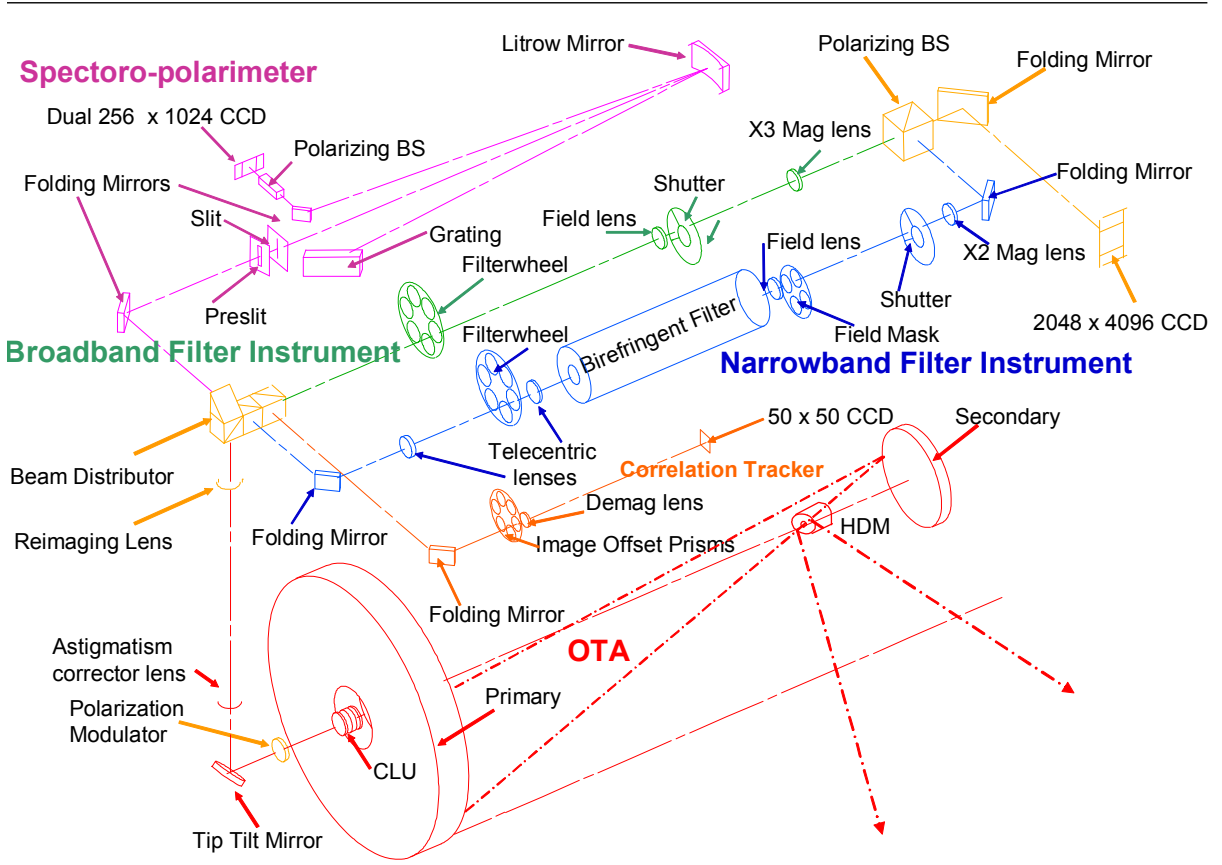

Fig. 8 The optical layout of the Hinode SOT identifies the five major optical systems with different colors. Of particular interest for this paper are the Narrowband Filter Instrument (blue) and the Spectro-Polarimeter (magenta), both of which are specifically designed to perform precision polarimetry for measurement of solar magnetic fields. For a detailed description of the Spectro-Polarimeter see Lites et al. (2013). Diagram taken from Tsuneta et al. (2008).

observations with the Spectro-Polarimeter (SP: Lites et al.2013) and filtergraphic observations with the Narrowband Filter Instrument (NFI). The spectrographic observations have the advantages that they provide measures of the spectral Stokes profiles simultaneously at all wavelengths, so that the integrity of the profiles in wavelength is retained, and also that solar events with high Doppler shift do not fall outside of the sampled wavelength range. These advantages are countered by the non-simultaneity of images of the solar scene that must be built up by stepping the narrow spectrograph slit across the image. One may have the best of both worlds with simultaneous filtergraphic and spectrographic Stokes polarimetry as implemented in the SOT/SP/NFI instrumentation.

The optical layout of the SOT Focal Plane Package (FPP) is shown in Fig. 8. Polarization modulation is accomplished by a rotating bi-crystalline retarder (quartz and sapphire) located in the symmetric part of the optical system just after the Optical Telescope Assembly (OTA). After the reflection by the tip-tilt mirror the beam is divided among four optical paths: the SP, the Broadband Filter Instrument (BFI), the NFI, and the Correlation Tracker. The NFI and BFI share a common focal plane and detector. The SP and NFI (or BFI) can operate simultaneously because the SP has its own detector. The polarization modulator rotates at $0.625 \mathrm{~Hz}$ such that the frame transfer detectors sample continuously at $10 \mathrm{~Hz}$ : a sampling speed fast enough to "freeze" moving features in the solar atmosphere for most polarimetric measurements. The SOT/SP operates in dual-beam mode by imaging both orthogonal linear polarizations exiting the polarizing beam splitter near its focal plane. Owing to continuous integration/readout of the detector, the instrument operates at close to $100 \%$ duty cycle. Several modes of polarimetry are available to the NFI, including frame-transfer modes using an 

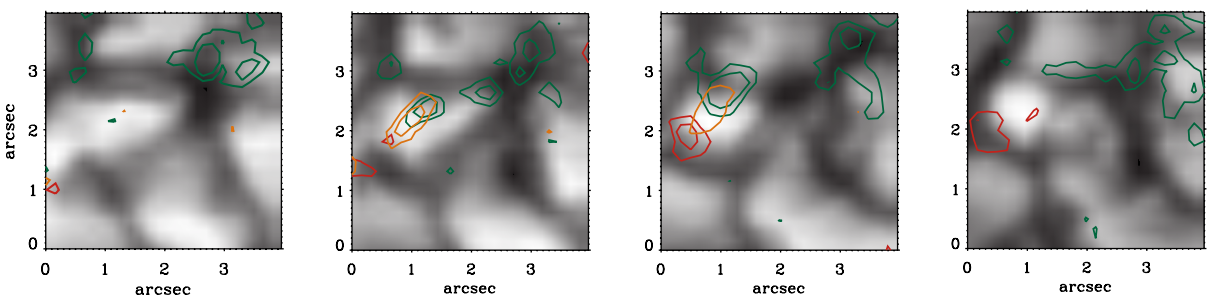

Fig. 9 This sequence of four continuum intensity images derived from repeated, short maps of the Hinode/SOT Spectro-Polarimeter reveal the emergence of a small dipole loop in a granule of the quiet Sun. Red and green contours represent positive and negative circular polarization (upward- and downward-directed magnetic field), and orange contours indicate linear polarization (horizontal field component). A loop structure is seen to form in the second frame with horizontal fields between the two vertically-oriented fields of opposite polarity. As the top of the loop rises above the photosphere, only vertical fields remain in the photosphere, each centered over a dark intergranular lane. Diagram taken from Centeno et al. (2007).

externally positioned mask of the focal plane. So both SOT/NFI and SOT/SP are capable of high precision polarimetry at high angular resolution.

Freedom from the disturbing effects of the Earth's atmosphere allows the Hinode polarimeters to continuously achieve angular resolution of the $50 \mathrm{~cm}$ SOT. This has permitted the detailed study of small-scale magnetism in the solar atmosphere. One area of intense study by Hinode researchers has been the magnetism of the quiet solar atmosphere, as illustrated by the example in Fig. 9 Prior to Hinode, glimpses from ground-based instrumentation revealed the presence of small-scale horizontal fields in the solar photosphere (Lites et al.1996), and it was suspected that they might correspond to emergence of small magnetic loops into the photosphere. With the advent of Hinode the widespread occurrence of these features was revealed for the first time (Lites et al. 2008) owing to the excellent image quality combined with high polarimetric precision. Using repeated short maps of quiet regions with SOT/SP, Centeno et al. (2007) were able to demonstrate that the horizontal fields are indeed the tops of tiny magnetic loops emerging within granules, as shown in Fig. 9

As a consequence of the seeing-free space environment, the point spread function (PSF) does not vary significantly during the time of an observation. This attribute allowed the development of inversion codes that take into account the PSF self-consistently during the minimization process, with the result that maps having diffraction-limited resolution of the SOT may be obtained for the physical parameters in the solar atmosphere. Such techniques are described in van Noort (2012); Asensio Ramos and de la Cruz Rodríguez (2015) and also in this special issue (de la Cruz Rodriguez et al. 2015). They have already resulted in numerous publications (e.g., Lagg et al. 2014; Tiwari et al. 2013, Buehler et al. 2015).

The Hinode mission was launched in September 2006 and still continues to operate providing measures of the photospheric magnetic field reliably and on demand. It is the only such space mission operating now, and comparable or better instrumentation is only on the horizon, being at least 6-10 years away. The SOT/SP is operating nominally, having experienced only a $25 \%$ drop in throughput over the past nine years on orbit. The SOT/NFI instrument continues to provide limited magnetogram data in the wing of the $\mathrm{Na} \mathrm{D}$-line only, owing to a progressive drift off-band of the pre-filters to the birefringent filter. Also, about a year into the mission, Hinode suffered a failure of its high-rate X-band telemetry. Fortunately, the lower rate S-band telemetry continued to function well, and conservative management of the data volume from SOT combined with a greatly increased number of downlink passes has allowed most of the SOT science objectives to continue to be addressed. 
This history of Hinode telemetry issues is yet another testament to the need for redundancy in space mission hardware.

\subsection{High-Altitude Balloon Missions}

Although they are not strictly space missions, a brief discussion of high-altitude balloon missions carrying instrumentation to measure the photospheric magnetic field is provided here. Balloon platforms reproduce some desirable conditions of space: 1) the rarified atmosphere at high altitude nearly completely eliminates the disruptive effect of atmospheric seeing, 2) during the high-latitude mid-summer extended periods of solar observing without night interruption are possible, and 3) some ultraviolet wavelengths become nearly transparent.

The cost of a balloon experiment is much smaller than that of an equivalent experiment in space because of relaxed restrictions on weight, power, contamination, vibration/acoustic tolerance, documentation, and other factors. Cost issues aside, development of a balloon mission is still a major undertaking. Taking the the two prior solar magnetographic balloon missions as typical, the development time for a major long-duration balloon experiment is comparable to that of a space mission. Reliability constraints of a balloon mission are similar to those of a space experiment since high altitude balloon launches are expensive and infrequent. One usually has the opportunity to recover the flight hardware after the end of a balloon mission, but that hardware must be able to survive a crash landing if it is to be flown again. In most respects, pointing and image stabilization of a balloon-borne high resolution telescope is more difficult than a space platform because one has to contend with variable winds at float altitude, and variable wind gradients between the balloon itself and its payload drive a pendulum motion.

Solar long-duration balloon experiments may be flown at mid-summer in the polar regions in order to have continuous observing throughout the flight. Two solar magnetographic experiments have been flown: the Flare Genesis Experiment (FGE, Bernasconi et al. 2000. 2001. 2002), and the successful Sunrise program as described in the following.

\subsubsection{Sunrise/IMaX}

The Sunrise program (Barthol et al. 2011) is a high-altitude, long-duration balloon experiment intended to explore solar phenomena at very high angular resolution (see also Kleint and Gandorfer 2015, in this issue). It was a joint effort of Germany, Spain, and the USA. It consisted of a $1.0 \mathrm{~m}$ telescope carrying the Imaging Magnetograph eXperiment (IMaX - Fig. 10, Martínez Pillet et al. 2011) and an ultraviolet imager (Sunrise Filter Imager (SuFI), Gandorfer et al. 2011). The former operated in the highly Zeeman-sensitive Fer line at $5250 \AA$ A. Also like FGE, IMaX used a pair of liquid crystal variable retarders to accomplish polarization modulation, but as a polarimeter IMaX had many features that qualified it as a quantitative solar polarimeter. IMaX measured combinations of four states that uniquely define the Stokes 4-vector. Compared to a polarimeter measuring pure states with six measurements, the IMaX scheme has significantly higher polarimetric efficiency (del Toro Iniesta and Collados 2000). Furthermore, the instrument had a nearly continuous expose/readout cycle resulting in a duty cycle of near unity. These features allowed IMaX to perform polarimetry at a $\mathrm{S} / \mathrm{N}$ appropriate for quantitative determination of the magnetic field vector. IMaX also used a tunable lithium niobate Fabry-Pérot filter in double pass so that it achieved a spectral resolution of $85 \mathrm{~m} \AA$ at $5250 \AA$. In typical full-Stokes polarimetry mode it sampled five wavelengths throughout the line profile, each with a $\mathrm{S} / \mathrm{N}$ of about 1000. 


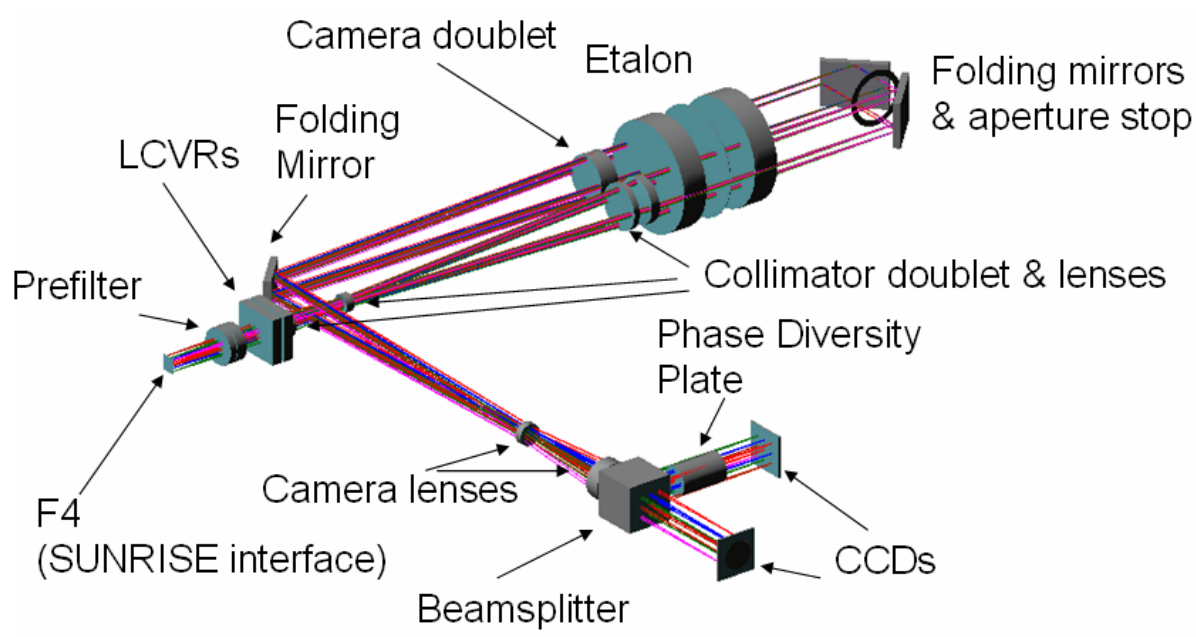

Fig. 10 The optical layout of the IMaX instrument on the Sunrise balloon program illustrates the simplicity of its design. Having two focal planes, it may operate as either a dual-beam polarimeter or, by applying a slight de-focus to one of the beams, as a phase-diversity imager. The wavelength isolation is accomplished by a tunable lithium niobate crystalline Fabry-Pérot etalon in double pass. Diagram taken from Martínez Pillet et al. (2011).

Two flights of Sunrise have been realized. The first flight in the north polar region lasted six days in June 2009, and produced outstanding results. High resolution sequences of data of duration up to 30 minutes were obtained of quiet Sun conditions. The Sunrise/IMaX system achieved close to its design angular resolution of about $0.15^{\prime \prime}$, i.e. a factor of two better than that of Hinode SOT/SP.

An example of the science that was made possible by Sunrise/IMaX is illustrated by Fig. 11 In that work, Martínez González et al. (2012) identify the presence of small-scale magnetic loops in the photosphere as evidenced by opposite circular polarization patches (upward- and downwardly-directed magnetic fields) connected by a detectable linear polarization patch between them (horizontal component of the field). Such loops were identified in short maps with the Hinode SOT/SP (see Fig. 9), but the high-resolution and highprecision imaging polarimetry of Sunrise/IMaX for the first time permitted this view of their spatial/temporal organization. This work reveals persistent areas where none of these smallscale loops appear: "dead calm" regions of the quiet Sun. The authors interpret this result - that the emergence of small-scale magnetic loops in the quiet Sun is not isotropic - as an important observed property of quiet Sun magnetism that must be explained by proposed mechanisms for its generation (such as a small-scale dynamo).

\subsection{Future Space Missions to Measure Solar Photospheric Magnetic Fields}

Perhaps there remain some hidden surprises regarding photospheric magnetic fields that are lurking below our current capability of resolving them because we have certainly not resolved the fine-scale structure of magnetism in the internetwork, and especially in the intergranular lanes. Both theory and simulations (e.g., Vögler and Schüssler 2007, Rempel 2014) and interpretation of observations (e.g., Pietarila Graham et al. 2009) suggest that observation of magnetic structure at the dominant scale of photospheric magnetism may be 

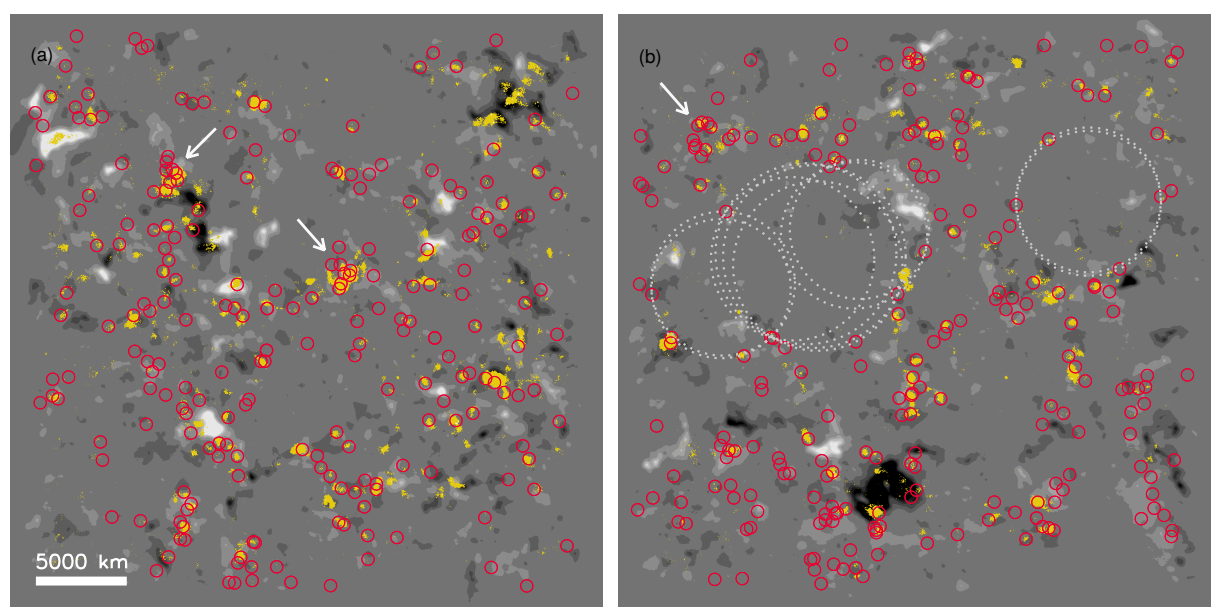

Fig. 11 Two sequences of polarimetric data from Sunrise/IMaX of durations 22 and 31 minutes are presented. Average longitudinal magnetograms are shown in greyscale. During these sequences, small-scale loops in the photosphere (red circles) appear as evidenced by linear polarization (yellow patches) separating two patches of opposite circular polarization. Dotted circles ("dead calm") in the image at right delimit areas where there is very little or no emergence of these small-scale loops. Arrows point to regions where several loops appear at the same location in the time series. Figure taken from Martínez González et al. (2012).

beyond reach with current diagnostic techniques, in recognition of the scattering properties of spectral lines in the photosphere when sampling scales at or below the photon mean free path. In any event, improvement of adaptive optical systems for the next generation of large ground-based telescopes is proceeding at a rapid pace, so future exploration of photospheric magnetism at very small scales would appear to be most aptly addressed using those facilities.

How then could future space observations contribute uniquely to further the understanding of photospheric magnetism? Little is still known regarding details of the photospheric field at the extreme solar poles. The polar regions are important in that it is believed that they play a crucial role in the progression of the large-scale solar dynamo. Magnetism of the polar regions has been studied extensively using Hinode (Ito et al. 2010; Shiota et al. 2012, and Petrie and Ettinger, this issue), but spatial resolution of the extreme poles is limited. Vertical fields are detected mainly by the transverse Zeeman effect and is therefore not very sensitive to weaker fields, and one is detecting fields well above the physical height corresponding to the $\tau_{c}=1$ level at disk center because of foreshortening. The Solar Orbiter mission will fly the Polarimetric and Helioseismic Imager (PHI) in an inclined orbit that will pass within 0.28 AU of the Sun (Kleint and Gandorfer 2015, this issue). PHI will perform precision polarimetry of the polar regions near closest approach, so magnetic fields of the polar regions will be attained from a more favorable viewpoint, albeit only for a brief period.

A new frontier for observational studies of solar magnetism is the chromosphere (see Sec. 57. Solar-C is an initiative for a major space mission lead by Japan but, like Hinode, having significant contributions from NASA and ESA. The major scientific thrust for Solar-C is the magnetism of the chromosphere, but interpretation of chromospheric field measurements will require accompanying measurements of fields in the photosphere. The Solar-C concept provides for simultaneous measurements of the field vector using spectral lines forming at several heights from the photosphere through the chromosphere. 
In the near term, it is extremely important for the discipline to continue to exploit its excellent resources for photospheric field measurement that are still on orbit. The community should strive to ensure that SDO/HMI and Hinode SOT/SP are operational as long as possible in order to build a synoptic data base of vector magnetic fields through a full solar cycle. One important motivation to sustain these observations is to understand the long-term behavior of non-AR fields: the remnant fields of active regions and also fields apparently not associated with the global dynamo. This is especially important if the most recent extended solar minimum and the present weak maximum heralds a major shift in the dynamo activity, for example the beginning of a new Maunder minimum.

\section{Chromospheric Magnetic Field Measurements}

5.1 The Importance of the Chromospheric Field Measurement to the Current Status of Solar Physics

The previous Sections 3 and 4 demonstrated the high level of sophistication reached especially for the measuremen 11 of the magnetic field in the photosphere, achieved by using advanced instrumentation in ground-based, balloon-borne and space-based observatories. The spatial and temporal resolution of the measurements in this deepest layer of the solar atmosphere directly accessible by observations has been increased by an order of magnitude within the last three decades. Similarly, recent space observatories operating at wavelengths not accessible from the ground permitted exploitation of the structure of the coronal magnetic field by analyzing the plasma emission of ions trapped in the coronal magnetic field with unprecedented precision.

The chromosphere is the coupling element between the photosphere and the corona. This extremely dynamic layer is located only one convection-cell size above the solar photosphere $(\approx 1000 \mathrm{~km})$. Exposed to the cold environment of space, the radiative energy losses effectively cool down the chromosphere. To maintain the typical temperature of approximately $10000 \mathrm{~K}$ a substantial amount of energy is required, about 15 times higher (Aschwanden et al. 2007) than the energy necessary to heat the solar corona. Whereas the "coronal heating problem" has attracted the attention of the solar physics community for decades, the equally important and interesting problem for the chromosphere has only very recently been emphasized. Similarly to the corona, the candidates for the chromospheric heating are wave dissipation, magnetic reconnection and Ohmic dissipation. Not only are the details of the underlying heating mechanisms poorly understood, but even the relative importance of the possible mechanisms in various structures remains poorly determined. The investigation of the chromospheric magnetism is crucial for both the understanding of the Sun-Earth connection with all its consequences to our technical and natural environment, and the detailed study of fundamental plasma physical processes in density and temperature regimes difficult to achieve in laboratories.

Progress in this field of research has been hindered by several aspects of inferring the properties of the chromosphere. The low gas and plasma densities result in large mean free paths for photons. In this radiatively-dominated regime the population of atomic levels deviates strongly from thermodynamic equilibrium, requiring complex and computationally expensive three-dimensional radiative transfer modeling to interpret the observations. Of

\footnotetext{
1 In this section, the term measurement refers to the determination of the magnetic field from remote sensing instruments by interpreting signals directly influenced by the solar magnetic field.
} 
similar complexity are magneto-hydrodynamic simulations, which for the photosphere have in the meantime become a reliable and robust tool to understand and analyze the fundamental physical processes. The basis to shed light into this complex field of research are reliable measurements of the chromospheric magnetic field at high spatial and temporal resolution.

\subsection{Challenges to High-Resolution Measurements of Chromospheric Fields}

The chromosphere "hides" itself between the observationally easier accessible layers photosphere and corona. With an average height of 500 to $2000 \mathrm{~km}$ above the solar surface it may be observed almost exclusively on the solar disk, with the consequence that the chromospheric information contained in the solar spectrum is to a large extent overwritten by the photospheric emission. The pressure scale height in the solar atmosphere is approximately $150 \mathrm{~km}$, leading to typical densities in the chromosphere of about four orders of magnitude lower than the photospheric ones. This decrease of gas pressure leads to an expansion and subsequently weakening of the magnetic field, rooted in often sub-arcsecond sized magnetic patches in the quiet-Sun photosphere.

The main tool for diagnosing the chromospheric magnetic field is spectropolarimetry in Fraunhofer lines formed under chromospheric conditions. With a few exceptions, the chromospheric signature in these spectral lines is only present in a narrow range around the line core. Weak fields and the low densities require highly sensitive spectropolarimetric measurements to detect these signals. Additionally, these low densities result in an increase of the typical velocities in the chromosphere: the Alfén speed reaches values of $100 \mathrm{~km} \mathrm{~s}^{-1}$, the sound speed is around $20 \mathrm{~km} \mathrm{~s}^{-1}$. Gas, plasma and wave motions can easily achieve these velocities, shock waves can form and reconnection of magnetic field lines can occur, making measurements at high temporal and spatial resolution mandatory.

The resulting dilemma was already discussed in Sec. 2 for photospheric conditions. There, the typical velocities are on the order of $5 \mathrm{~km} \mathrm{~s}^{-1}$, but the velocities in the chromosphere can easily be an order of magnitude higher. As a consequence, the maximum permissible exposure time to freeze in the solar evolution for a large aperture solar telescope $(\gtrsim 1 \mathrm{~m})$ lies in the ten millisecond range (see Fig. 117). On the other hand, the photon flux in the line core, i.e., the part of the spectrum containing the chromospheric information, is on the average only 10-20\% of the continuum intensity. Additionally, to characterize the weak chromospheric fields, a high $\mathrm{S} / \mathrm{N}$ ratio in the range of a few times $10^{-4}$ is desirable. To achieve these goals, highly photon efficient instrumentation on large aperture solar telescopes, not necessarily operated at their diffraction limit, is mandatory. The Daniel K. Inouye Solar Telescope (DKIST, Rimmele and ATST Team 2008; Tritschler et al. 2015), currently under construction on Maui/Hawaii, and the European Solar Telescope (EST, Collados et al. 2013) are the logical steps in this direction.

Besides these technical challenges, chromospheric conditions introduce a high level of complexity to the interpretation of the polarization signals. Simplifying assumptions, like the local thermodynamic equilibrium (LTE) conditions prevailing in the photosphere, do not hold anymore. Chromospheric absorption and emission processes need to be modeled under non-LTE conditions involving on average 6-12 atomic levels in the most prominent atoms used for chromospheric diagnostics $(\mathrm{H}, \mathrm{Ca}, \mathrm{Mg}$, and $\mathrm{He})$. The population of the levels in these atoms additionally strongly depends on the incident coronal radiation field. Its anisotropy in the illumination of the chromospheric layer is responsible for creating imbalances in the population of the sub-levels of the atoms, leading to atomic polarization. Long time scales of up to several hours for ionization and recombination processes introduce a 
memory effect of the chromosphere to past events, complicating the determination of the initial conditions for theoretical models (de la Cruz Rodriguez et al. 2015, this issue).

\subsection{Diagnostic Techniques}

Our knowledge about the chromospheric magnetic field is mainly based on the interpretation of the polarimetric signal in chromospheric spectral lines. The above mentioned difficulties in measuring the polarimetric signal in these lines motivates the search for alternative diagnostic techniques. Two possible candidates are: extrapolations based on photospheric magnetic field maps and measurements in the millimeter and sub-millimeter wavelength range, soon available at high spatial resolution using the ALMA (Atacama Large Millimeter/submillimeter Array).

Extrapolations of the photospheric field rely on the robustness and simplicity of photospheric magnetic field measurements, which can be obtained on a routine basis at higher spatial and temporal resolution than chromospheric measurements. During the past decade a significant improvement on the reliability of these extrapolations has been achieved, for example by including $\mathrm{H} \alpha$ images in the pre-processing of the data (Wiegelmann et al.2008). Unfortunately, the principle of extrapolations, based on minimizing the complexity of the magnetic field in the observed atmospheric volume, is not applicable for the most interesting and very dynamic conditions of, e.g., shocks or reconnection sites. To uncover the secrets at these locations, direct measurements are mandatory.

The linear relation between millimeter wavelength emissions and the chromospheric temperature makes ALMA an ideal thermometer for the solar chromosphere. In addition, in the near future it will perform magnetic field measurements in the chromosphere with a spatial resolution of down to $0.2^{\prime \prime}$ based on two physical processes: the Zeeman effect in high-n recombination lines of hydrogen and diatomic molecules, and the influence of the magnetic field to the temperature distribution by suppressing the power of propagating waves. Details about the usage of ALMA for chromospheric diagnostics can be found in this issue (Judge et al. 2015) and in Wedemeyer et al. (2015).

In the following sections we return to the current state-of-the-art measurement technique for chromospheric fields using spectropolarimetry in Zeeman and Hanle sensitive spectral lines in the visible and the near infrared regime. Here the improved observational capabilities have led to significant progress during the last couple of years, which will continue in the coming decade with the advent of large-aperture solar telescopes.

\subsubsection{Diagnostic Spectral Lines}

In the strictest sense the term chromosphere is defined as the region of $\mathrm{H} \alpha$ emission. Using the $\mathrm{H} \alpha$ line as the tool to investigate the chromospheric magnetism therefore seems to be an obvious choice. Unfortunately, the above-mentioned difficulties in modeling spectral lines are most prominent in this line. $\mathrm{H} \alpha$ originates from an excited level of the hydrogen atom $(n=3)$, whose population strongly depends on the temperature and radiative processes in the atmosphere, and therefore making the interpretation of the spectral profiles exceptionally complex (Leenaarts et al. 2012).

Spectral lines that are easier to interpret have been identified and are now observed on a routine basis. Fig. 12 (snapshot from Bifrost chromospheric modeling code, taken from Carlsson et al. 2015) presents an overview of these lines which have emerged as especially well suited for diagnosing chromospheric magnetism. Sorted by their formation height from 


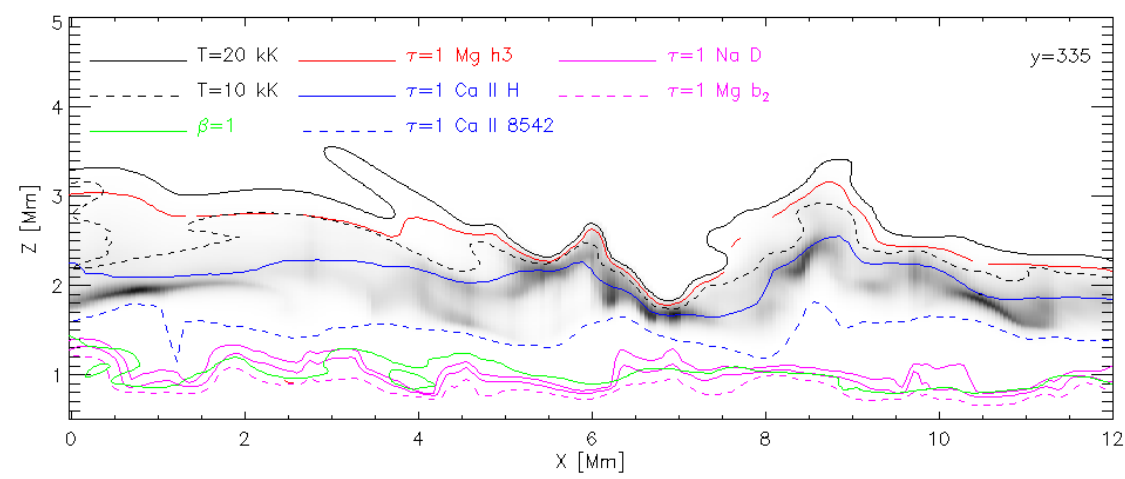

Fig. 12 Formation heights of various chromospheric spectral lines computed from the self-consistent chromospheric modeling code Bifrost (Gudiksen et al. 2011). The snapshot from a time series shows the isotemperature surfaces (black lines), the plasma- $\beta=1$ layer (green) and the optical depth $\tau=1$ layers for the spectral lines as labeled in the figure. The layer of absorption in the He I $1083 \mathrm{~nm}$ triplet is indicated by the gray-shaded area. Taken from Carlsson et al. (2015).

bottom to top, obtained from non-LTE modeling, these lines are: $\mathrm{Mg}_{\mathrm{I}} \mathrm{B}_{2}$ at $517 \mathrm{~nm}$, the $\mathrm{Na}$ D line pair at $589 \mathrm{~nm}$, the Ca II infrared triplet at $854 \mathrm{~nm}$, the Ca II H\&K lines at $390 \mathrm{~nm}$, $\mathrm{He}_{\mathrm{I}} \mathrm{D} 3$ at $588 \mathrm{~nm}$, the $\mathrm{He}_{\mathrm{I}}$ triplet at $1083 \mathrm{~nm}$, and the $\mathrm{Mg}$ II h\&k lines at $279 \mathrm{~nm}$. The latter has been observed on a routine basis only in spectroscopic and imaging mode by the IRIS mission (De Pontieu et al. 2014) and the second Sunrise stratospheric balloon flight (Barthol et al. 2011). Its potential to retrieve the magnetic field vector is very high, but so far limited to theoretical calculations (Trujillo Bueno 2014).

The quiet-Sun modeling underlying Fig. 12 demonstrates the high corrugation of especially the upper chromospheric layers (Carlsson et al. 2015). The corrugation becomes even stronger above active regions containing pores or sunspots. The height variation can easily span several $\mathrm{Mm}$ and must be taken into account for the correct interpretation of the spectropolarimetric signals.

The Ca II and He I infrared triplets are currently in the focus for the diagnostics of chromospheric magnetism. The analysis of the Ca II lines has benefited from improvements in the computationally expensive non-LTE modeling (e.g., Leenaarts et al. 2009, de la Cruz Rodríguez et al. 2012) and the availability of image reconstruction techniques, thereby permitting production of polarimetric maps at the diffraction limit of the modern solar telescopes at high signal-to-noise ratio (Scharmer 2006, Cavallini|2006, Puschmann et al. 2012). Inversions of the radiative transfer equation (RTE) to retrieve the physical conditions in the chromosphere from the measured Stokes spectra are on a good route to become a standard tool for the solar community, similarly to the inversion tools currently available for the photosphere. The progress in this field is summarized in this issue in the article (de la Cruz Rodriguez et al. 2015, this issue).

\subsubsection{The He I $1083 \mathrm{~nm}$ Triplet}

This triplet has gained attraction due to two major improvements: developments in detector technology allow for spectropolarimetric observations at noise levels down to the $10^{-4}$ range (in units of the continuum), and the theoretical explanation for the linear polarization signal in the absence of magnetic fields (Trujillo Bueno et al. 2002). The latter led to the 
development of easy-to-use inversion codes (Asensio Ramos et al. 2008, Lagg et al. 2009). The biggest advantage of the He I $1083 \mathrm{~nm}$ line over other chromospheric lines is the absence of almost any photospheric contamination in the signal. The reason for this lies in the special formation process of this triplet. The He I $1083 \mathrm{~nm}$ line (as well as the He ID3 line) is a transition in the triplet state of the He atom, which is only sparsely populated under normal photospheric and chromospheric conditions. Coronal ultra-violet (UV) radiation with a wavelength shorter than $50.4 \mathrm{~nm}$ (i.e., $24.58 \mathrm{eV}$ ) is required to ionize the He atoms. The subsequent recombination occurs with equal probability either back to the singlet state, or to the triplet state. Since the chromosphere is highly opaque to coronal UV radiation, this ionization process occurs only in the top most layer of the chromosphere, resulting in a thin slab containing He $\mathrm{I}$ atoms in the triplet state. To first order, the physical conditions in this thin slab are height-invariant, an assumption validated by the simplicity of the observed Zeeman signals in this triplet. The flip side of this special formation process is the weak absorption signature above quiet Sun regions, where coronal UV illumination is very low.

\subsubsection{He $1083 \mathrm{~nm}$ and the Hanle Effect}

A further strength of the He I triplet is the coverage of a wide range of magnetic field strengths: The Landé factors of the He I triplet $\left(g_{\text {eff }}=2.0,1.75,1.25\right)$ allow for reliable measurements of the magnetic field vector above $\approx 50-100 \mathrm{G}$ using the Zeeman effect. For higher field strengths the Paschen-Back effect sets in, shifting the Zeeman sub-levels and introducing an asymmetry in the Stokes profiles (Socas-Navarro et al. 2004, 2005; Sasso et al. 2006). The Hanle effect covers the region of lower field strengths (0.1-100 G, Trujillo Bueno et al. 2002). Anisotropic illumination of the thin slab by the photospheric radiation field introduces imbalances in the population of the lower and upper levels of the transition, producing a characteristic linear polarization signal. Weak magnetic fields of up to $\approx 8 \mathrm{G}$ change the strength and the direction of polarization (Hanle sensitive regime), whereas the linear polarization signals for stronger fields are only sensitive to the field direction (Hanle saturated regime).

Despite the above-mentioned advantages of the He triplet, the correct interpretation of the signals poses major challenges for the near future. For the correct modeling of the strength of the line, the coronal environment must be taken into account as well as the importance of collisions for the population of the triplet state. The Hanle measurements require an exact determination of the anisotropy of the incident radiation field, influenced not only by the height of the slab, but also by the presence of brightness variations in the photosphere (e.g. sunspots), and the absorption of optically thick He I layers. Additionally, the ambiguities introduced by the Hanle effect must be treated carefully.

The near-infrared spectral region is particularly well suited for the large-aperture groundbased telescopes which were commissioned recently. The reasons are better and more stable seeing (Turon and Léna 1970), less scattered light (Staveland 1970) and less atmospheric extinction. The New Solar Telescope (NST, Goode et al. 2010) at the Big Bear Solar Observatory with an aperture of $1.6 \mathrm{~m}$ and the GREGOR Telescope on Tenerife $(1.5 \mathrm{~m}$, Schmidt et al. 2012) are both equipped with instrumentation for magnetic field diagnostics in the near infrared region, with one of the main foci being on imaging polarimetry and spectropolarimetry in the He I 1083 m triplet. 

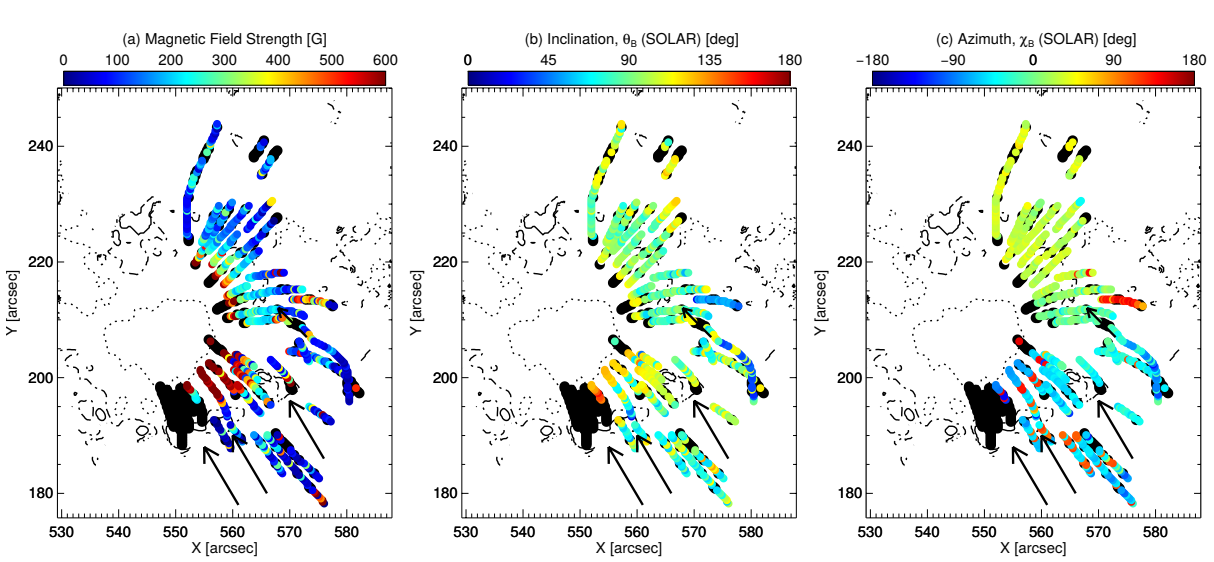

Fig. 13 Magnetic field strength, inclination and azimuth (in solar reference frame) of superpenumbral fibrils observed in the He I $1083 \mathrm{~nm}$ triplet with FIRS at the DST on January 292012 . The central part of the filament is characterized by horizontal fields below $300 \mathrm{G}$, well aligned to the visible fibrils (from Schad 2013).

\subsection{Examples of Current Chromospheric Field Measurement Results}

\subsubsection{He I $1083 \mathrm{~nm}$ Triplet}

Two examples of recent He I $1083 \mathrm{~nm}$ observations were selected to demonstrate recent advances in the interpretation of the spectropolarimetric data and the progress in instrumentation, allowing for observations at unprecedented spatial resolution and polarimetric sensitivity in this line. The first one shows data from the Facility Infrared Spectropolarimeter (FIRS, Jaeggli et al.2010) at the National Solar Observatory's Dunn Solar Telescope (DST): Schad (2013) presents an observation of superpenumbral chromospheric fibrils spanning from the penumbra of NOAA AR 11408 to the internetwork regions outside the active region (see Fig. 13. Limited by the diffraction limit of the instrument of $0.6^{\prime \prime}$, full Stokes maps in He I $1083 \mathrm{~nm}$ were recorded with an average noise level of $3-4 \times 10^{-4}$ in units of the continuum intensity. The spectra were analyzed with the inversion code HAZEL ("Hanle and Zeeman Light", Asensio Ramos et al. 2008) which is based on the multiterm calculations of the orthohelium atom of Landi Degl'Innocenti and Landolfi (2004). Schad focussed their analysis on the determination of the magnetic field orientation in these fibrils to answer the question whether the magnetic field vector is aligned with the structures observed simultaneously in $\mathrm{H} \alpha$ and $\mathrm{Ca}$ II infrared intensity maps. This required a thorough analysis to select the correct solution out of up to 8 ambiguous solutions, a consequence of the combination of the well known $180^{\circ}$ ambiguity of Zeeman effect with the so called Van Vleck ambiguity (see, e.g., Casini et al. 2005, 2009, Merenda et al. 2006, Orozco Suárez et al. 2015).

As a result of this analysis, Schad (2013) concluded that the projected direction of the field derived from $\mathrm{He}_{\mathrm{I}}$ inversions does not deviate by more than $\pm 10^{\circ}$ from the visible fibrils. For most of the fibrils observed in the Ca II $854.2 \mathrm{~nm}$ line, de la Cruz Rodríguez and SocasNavarro (2011) obtained a similar alignment, but in some of the Ca II fibrils the magnetic field direction deviates significantly from the visible structures. A thorough investigation of more fibrils at high spatial resolution, ideally simultaneously in $\mathrm{He}_{\mathrm{I}}$ and $\mathrm{Ca}$ II, is required to resolve this discrepancy.

The largest European solar telescope, GREGOR, entered the scientific phase in 2014 (Schmidt et al.2012). The GREGOR Infrared Spectrograph (GRIS, Collados et al. 2012) is 

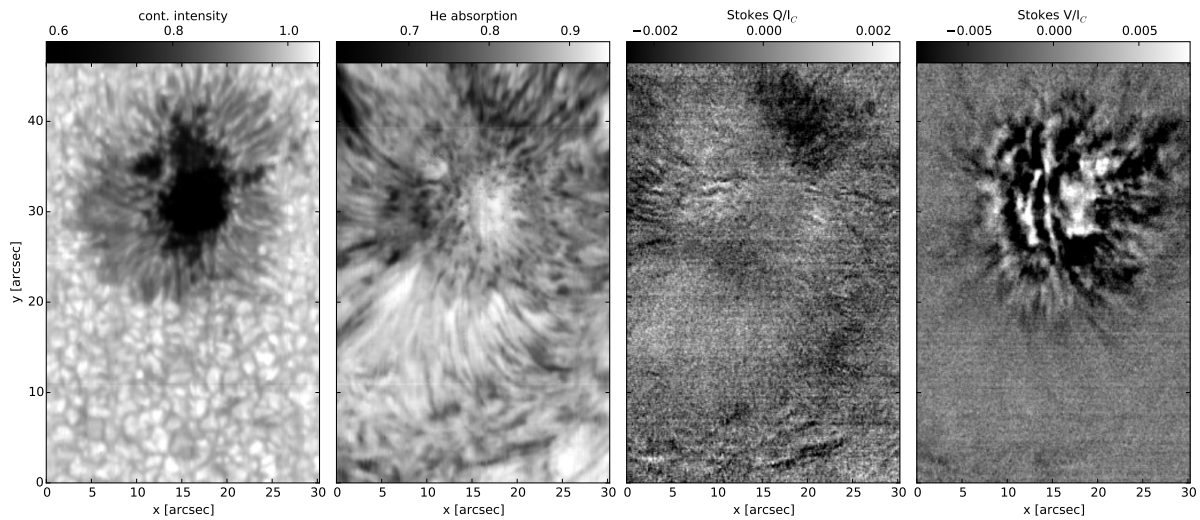

Fig. 14 Continuum image and He I $1083 \mathrm{~nm}$ Stokes $I, O$, and $V$ maps of AR 12096 obtained with GREGOR/GRIS on June 272014 close to disk center $(\mu=0.98)$. The achieved spatial resolution is $0.44^{\prime \prime}$ at a spectral resolution of 200000 . The total exposure time of $1.6 \mathrm{~s}$ per slit position for the full Stokes vector (without readout and processing time) resulted in a noise level of $7-10 \times 10^{-4}$ of the continuum intensity.
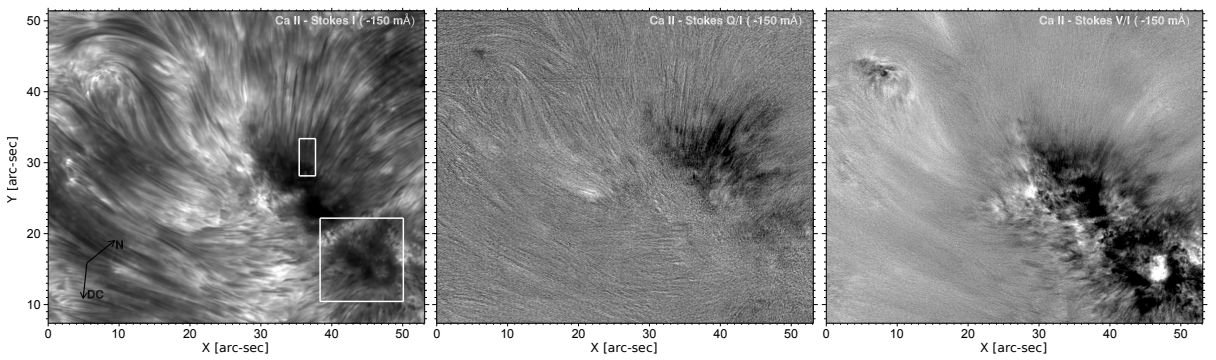

Fig. 15 CRISP Stokes $I, Q / I$, and $V / I$ filtergrams at $\Delta \lambda=-150 \mathrm{~m} \AA$ from the core of the Ca II $854.2 \mathrm{~nm}$ line (adapted from de la Cruz Rodríguez et al. 2013). The boxes mark the regions for which a detailed analysis of umbral flashes and running penumbral waves was performed.

producing spectropolarimetric $\mathrm{He}$ I $1083 \mathrm{~nm}$ data with an unprecedented spatial resolution close to the diffraction limit of the telescope at signal to noise ratios in the Stokes parameters of up to 3000. An example of such Stokes parameter maps, composed from the scan of the slit over AR 12096 on June 27 2014, is presented in Fig. 14. A detailed analysis of the fine structure of the chromospheric field is currently being performed at the partner institutions (Kiepenheuer Institut für Sonnenphysik, Freiburg; Max-Planck-Institut für Sonnensystemforschung, Göttingen; Leibniz-Institut für Astrophysik, Potsdam; Instituto de Astrofísica de Canarias, La Laguna) and will soon appear in a peer-reviewed journal.

\section{$5.4 .2 \mathrm{Ca}$ II $854.2 \mathrm{~nm}$}

The highest spatial resolution for spectropolarimetric measurements is currently achievable only using filter-based instruments. As described in Sec. 3.2.1 these instruments allow to apply image reconstruction routines to fully exploit solar telescopes down to their theoretical resolution limits. The most successful instruments of this type are the CRisp Imaging SpectroPolarimeter at the 1-meter Swedish Solar Telescope (SST/CRISP, Scharmer et al. 2008) and the Interferometric BIdimensional Spectrometer (IBIS, Cavallini 2006). 
An example of a chromospheric magnetic field measurement with CRISP is presented in Fig. 15. showing the Stokes I, Q/I, and V/I map of AR 11204 observed on May 42011. de la Cruz Rodríguez et al. (2013) applied the non-LTE inversion code NICOLE (SocasNavarro et al. 2000) to this dataset and obtained the height dependence of temperature, line-of-sight velocity and magnetic field vector above the umbra and the penumbra of the sunspot at a spatial resolution of $\approx 0.15^{\prime \prime}$. The high temporal cadence of CRISP ( $16 \mathrm{~s}$ for a full-Stokes line scan) enabled the authors to perform a detailed analysis of umbral flashes, revealing their $\approx 1000 \mathrm{~K}$ hotter shock front when compared to the surrounding material, without a measurable influence on the magnetic field strength and direction. Additionally, a surprisingly large variation of the magnetic field strength (up to $200 \mathrm{G}$ ) in the chromosphere above the penumbra could be attributed to running penumbral waves. For more examples of high-quality measurements in the Ca II line using CRISP and IBIS data the reader is referred to (Pietarila et al.2007) and (Kleint 2012).

\section{Technology and Data Analysis Challenges}

The previous chapters demonstrated that the determination of the magnetic field vector using remote sensing instruments requires a close intermeshing of observational techniques and analysis methods. Technology must be pushed to its limits to obtain data sets with high $\mathrm{S} / \mathrm{N}$ ratios in all Stokes parameters. An accurate knowledge of all instrumental deficiencies, unavoidably resulting in data degradation, is necessary in order to treat them carefully in the data analysis pipelines. The most common tool for extracting the magnetic field from the observations is the inversion of the Stokes spectra: From an initial guess model atmosphere synthetic Stokes spectra are computed, then the parameters of this model atmosphere are adjusted iteratively until the synthetic Stokes spectra agree with the observed ones (see also de la Cruz Rodriguez et al. 2015 this issue). In this process it is extremely important that the synthetic Stokes spectra are degraded in exactly the same way as the instrument alters the spectra originating from the Sun. Only then the comparison between the observed and synthetic Stokes profile will deliver reliable information about the conditions in the solar atmosphere.

The aim is, of course, to keep the instrumental deficiencies as small as possible. This is where technological improvements come into play, which have allowed for a significant increase in the accuracy of spectro-polarimetric measurements at high spatial resolution during the last decade. The key ingredients here are advances in detector technology (e.g. FSP, see Sec. 3.5), massively multiplexed focal planes (2-dimensional spectropolarimeters, see Sec. 3.2.2, and the use of adaptive optic systems mandatory for operating large aperture solar telescopes at their diffraction limit (see Sec. 3.3.1.

In the near future, large-aperture solar telescopes will allow to observe solar features well below scales of $50 \mathrm{~km}$. Another challenge for the future is to improve the height resolution by performing spectropolarimetric measurements in many spectral lines simultaneously. The difference in the formation height of the lines and in the response to changes of atmospheric parameters will help modern inversion tools to retrieve the stratification in the solar atmosphere with unprecedented accuracy.

Despite all the efforts to avoid instrumental deficiencies, they will always be present in the data. The proper treatment of these instrumental effects is a big challenge on the data analysis side. This has been done successfully during the last years in terms of image reconstruction techniques based on phase-diversity, speckle, or MOMFBD techniques (Lofdahl and Scharmer 1994, von der Luehe 1993, van Noort et al. 2005). The next evolutionary 
steps in this direction are novel inversion techniques, like the spatially-coupled inversions by van Noort (2012) or the sparse inversion of Stokes profiles by Asensio Ramos and de la Cruz Rodríguez (2015), which take into account the point-spread-function (PSF) of the telescope self-consistently during the inversion process. In a next development step, inversion programs must take into account the natural 2D-coupling between the single-column atmospheres.

But even the cleanest data are subject to ambiguous solutions in the determination of the physical conditions in the solar atmosphere. Such ambiguities are caused by the physical process producing the measured signal (e.g., the well-known $180^{\circ}$ ambiguity of the Zeeman effect, or the Van Vleck ambiguity in the Hanle diagnostics), or by the mere presence of a multitude of model atmospheres producing the same Stokes vector within the noise level of the data (degeneracy of the fit parameters).

The resolution of ambiguities is a complex and very often not unique process, involving assumptions about the physical conditions in the solar atmosphere. A successful technique to solve for these ambiguities is to prescribe certain conditions to the solar atmosphere, for example minimizing the divergence of the magnetic field or the vertical current density. A description about available tools for removing the $180^{\circ}$ Zeeman ambiguity was given by Metcalf et al. (2006). The solution of the so-called Van Vleck ambiguity, present in Hanle measurements, often requires to choose the "more likely" solution, i.e., the solution matching a realistic, physical model of the observed structure (e.g., Orozco Suárez et al. 2014, Schad et al. 2013).

The identification of the degeneracy in the solution is another important step towards the reliable interpretation of spectropolarimetric data. It helps to identify the simplest model atmosphere compatible with the observed Stokes spectra, which is usually the one with the lowest number of free parameters. Bayesian inversion techniques (Asensio Ramos et al. 2007) or Markov chain Monte Carlo (MCMC) methods are promising approaches to detect degeneracies.

Ideally, ambiguities and degeneracies in the solutions should be addressed directly by the observations: multi-line spectropolarimetry can provide the complementary information needed to find the unique solution. Similarly, observations from different vantage points are beneficial. The latter will be achieved for the first time by the out-of-ecliptic mission Solar Orbiter (see Sec. 4), which will provide high-resolution Stokes maps of the photosphere with the Polarimetric and Helioseismic Imager (PHI).

\section{Conclusions}

As mentioned in the introduction, the Sun would be quietly evolving along the main sequence if it were not for its magnetic field. Solar magnetism is responsible for solar activity and space weather events, some of which affect our modern civilization. Magnetic processes appear to be ubiquitous throughout the universe. So for both astrophysical and practical reasons there has been increasing interest in observing and understanding the solar magnetic field. This increase is suggested by Fig. 16 which shows 5-year totals of papers containing "solar or sun" and "photosphere or chromosphere" and "magnetic" in either the title or the abstract of refereed journals (based on NASA ADS). Even more impressive is the increase in the percentage of the publications relative to the ones without the search tag "magnetic": during the last 5 years, more than $60 \%$ of all papers on the solar photosphere and chromosphere deal with the subject magnetism. 


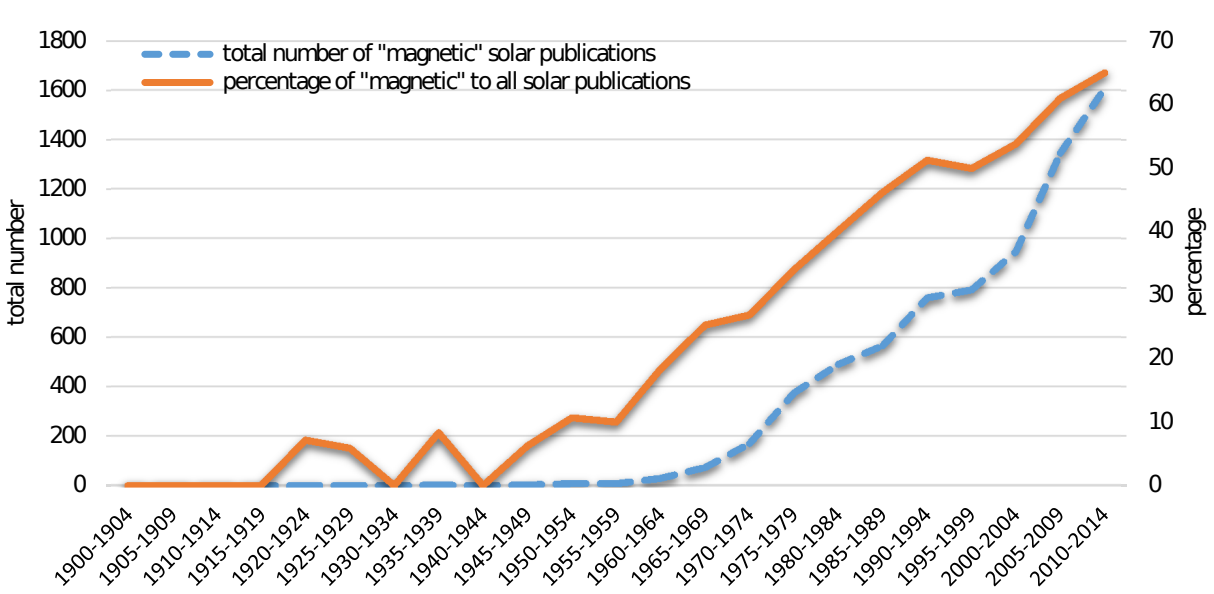

Fig. 16 5-year totals of papers published in refereed journals including the words "(solar OR sun) AND (photosphere OR chromosphere) AND magnetic" in the title or abstract according to ADS (dashed blue curve). The solid orange curve indicates the percentage of these publications relative to the ones without the search tag "magnetic" (right ordinate axis).

This impressive growth of interest in solar magnetism stems to a large extent from the recognition of its importance to almost all fundamental physical processes on the Sun and its relevance to space weather, including the efforts to predict space weather events. The rapid development of measurement techniques for solar magnetic fields is a logical consequence, additionally supported by progress in technology, especially in the field of detectors and optical instrumentation. Together with the recent and forthcoming introduction of new telescopes we expect a steady increase of new measurements and understanding of the solar magnetic field. Recent excitement in exoplanet discovery and research has awakened interest in new techniques of spectropolarimetry of stars that should benefit solar observational methods. In the entire two-century history of solar polarimetry, the next decade should be the most exciting and scientifically productive.

Acknowledgements Hinode is a Japanese mission developed and launched by ISAS/JAXA, collaborating with NAOJ as a domestic partner, NASA and STFC (UK) as international partners. Scientific operation of the Hinode mission is conducted by the Hinode science team organized at ISAS/JAXA. This team mainly consists of scientists from institutes in the partner countries. Support for the post-launch operation is provided by JAXA and NAOJ (Japan), STFC (UK), NASA, ESA, and NSC (Norway). B. Lites was supported in part by the FPP project at LMSAL and HAO under NASA contract NNM07AA01C. The National Center for Atmospheric Research is sponsored by the National Science Foundation. The National Solar Observatory is operated by the Association of Universities for Research in Astronomy (AURA, Inc.) under a cooperative agreement with the National Science Foundation. The 1.5-meter GREGOR solar telescope was built by a German consortium under the leadership of the Kiepenheuer-Institut für Sonnenphysik in Freiburg with the Leibniz-Institut für Astrophysik Potsdam, the Institut für Astrophysik Göttingen, and the Max-Planck-Institut für Sonnensystemforschung in Göttingen as partners, and with contributions by the Instituto de Astrofisica de Canarias and the Astronomical Institute of the Academy of Sciences of the Czech Republic. This study is supported by the European Commission's FP7 Capacities Programme under the Grant Agreement number 312495 .

\section{References}

M.J. Aschwanden, A. Winebarger, D. Tsiklauri, H. Peter, The Coronal Heating Paradox. Astrophys. J. 659, 
1673-1681 (2007). doi:10.1086/513070

A. Asensio Ramos, J. de la Cruz Rodríguez, Sparse inversion of Stokes profiles. I. Two-dimensional MilneEddington inversions. Astron. Astrophys. 577, 140 (2015). doi:10.1051/0004-6361/201425508

A. Asensio Ramos, M.J. Martínez González, J.A. Rubiño-Martín, Bayesian inversion of Stokes profiles. Astron. Astrophys. 476, 959-970 (2007). doi:10.1051/0004-6361:20078107

A. Asensio Ramos, J. Trujillo Bueno, E. Landi Degl'Innocenti, Advanced Forward Modeling and Inversion of Stokes Profiles Resulting from the Joint Action of the Hanle and Zeeman Effects. ApJ 683, 542-565 (2008). doi:10.1086/589433

H.W. Babcock, The Solar Magnetograph. Astrophys. J. 118, 387 (1953). doi:10.1086/145767

J.L. Ballester, Prominence Seismology, in IAU Symposium, ed. by B. Schmieder, J.-M. Malherbe, S.T. Wu IAU Symposium, vol. 300, 2014, pp. 30-39. doi:10.1017/S1743921313010703

P. Barthol, A. Gandorfer, S.K. Solanki, M. Schüssler, B. Chares, W. Curdt, W. Deutsch, A. Feller, D. Germerott, B. Grauf, K. Heerlein, J. Hirzberger, M. Kolleck, R. Meller, R. Müller, T.L. Riethmüller, G. Tomasch, M. Knölker, B.W. Lites, G. Card, D. Elmore, J. Fox, A. Lecinski, P. Nelson, R. Summers, A. Watt, V. Martínez Pillet, J.A. Bonet, W. Schmidt, T. Berkefeld, A.M. Title, V. Domingo, J.L. Gasent Blesa, J.C. Del Toro Iniesta, A. López Jiménez, A. Álvarez-Herrero, L. Sabau-Graziati, C. Widani, P. Haberler, K. Härtel, D. Kampf, T. Levin, I. Pérez Grande, A. Sanz-Andrés, E. Schmidt, The Sunrise Mission. Solar Phys. 268, 1-34 (2011). doi:10.1007/s11207-010-9662-9

P.N. Bernasconi, D.M. Rust, H.A.C. Eaton, High Resolution Vector Magnetograms with the Flare Genesis Vector Polarimeter, in Advanced Solar Polarimetry - Theory, Observation, and Instrumentation, ed. by M. Sigwarth Astronomical Society of the Pacific Conference Series, vol. 236, 2001, p. 399

P.N. Bernasconi, D.M. Rust, H.A. Eaton, G.A. Murphy, Balloon-borne telescope for high-resolution solar imaging and polarimetry, in Airborne Telescope Systems, ed. by R.K. Melugin, H.-P. Röser Society of Photo-Optical Instrumentation Engineers (SPIE) Conference Series, vol. 4014, 2000, pp. 214-225

P.N. Bernasconi, D.M. Rust, M.K. Georgoulis, B.J. Labonte, Moving Dipolar Features in an Emerging Flux Region. Solar Phys. 209, 119-139 (2002). doi:10.1023/A:1020943816174

F.H. Bigelow, The solar corona discussed by spherical harmonics. Contrib. Smithsonian Inst. 691, 22 (1889)

J.M. Borrero, P. Kobel, Inferring the magnetic field vector in the quiet Sun. III. Disk variation of the Stokes profiles and isotropism of the magnetic field. Astron. Astrophys. 550, 98 (2013). doi:10.1051/0004$6361 / 201118239$

I.S. Bowen, The Image-Slicer a Device for Reducing Loss of Light at Slit of Stellar Spectrograph. Astrophys. J. 88, 113 (1938). doi:10.1086/143964

T. Brown, The Fourier Tachometer: Principles of Operation and Current Status, in Solar instrumentation: What's next?, ed. by R.B. Dunn, 1981, p. 150

D. Buehler, A. Lagg, S.K. Solanki, M. van Noort, Properties of solar plage from a spatially coupled inversion of Hinode SP data. Astron. Astrophys. 576, 27 (2015). doi:10.1051/0004-6361/201424970

M. Carlsson, V.H. Hansteen, B.V. Gudiksen, J. Leenaarts, B. De Pontieu, A publicly available simulation of an enhanced network region of the Sun. ArXiv e-prints (2015)

R. Casini, R. Bevilacqua, A. López Ariste, Principal Component Analysis of the $\mathrm{He} \mathrm{I}_{3}$ Polarization Profiles from Solar Prominences. ApJ 622, 1265-1274 (2005). doi:10.1086/428283

R. Casini, A.G. de Wijn, P.G. Judge, Analysis of Seeing-induced Polarization Cross-talk and Modulation Scheme Performance. Astrophys. J. 757, 45 (2012). doi:10.1088/0004-637X/757/1/45

R. Casini, A. López Ariste, F. Paletou, L. Léger, Multi-Line Stokes Inversion for Prominence Magnetic-Field Diagnostics. ApJ 703, 114-120 (2009). doi:10.1088/0004-637X/703/1/114

F. Cavallini, IBIS: A New Post-Focus Instrument for Solar Imaging Spectroscopy. Solar Phys. 236, 415-439 (2006). doi:10.1007/s11207-006-0103-8

R. Centeno, H. Socas-Navarro, B. Lites, M. Kubo, Z. Frank, R. Shine, T. Tarbell, A. Title, K. Ichimoto, S. Tsuneta, Y. Katsukawa, Y. Suematsu, T. Shimizu, S. Nagata, Emergence of Small-Scale Magnetic Loops in the Quiet-Sun Internetwork. ApJL 666, 137-140 (2007). doi:10.1086/521726

W.C. Chaplin, Y. Elsworth, G.R. Isaak, R. Lines, C.P. McLeod, B.A. Miller, R. New, H.B. van der Raay, Observing the sun with the Birmingham Solar-Oscillations Network (BISON). The Observatory 116, 32-33 (1996)

M. Collados, A. Lagg, J.J. Díaz Garcí A, E. Hernández Suárez, R. López López, E. Páez Mañá, S.K. Solanki, Tenerife Infrared Polarimeter II, in The Physics of Chromospheric Plasmas, ed. by P. Heinzel, I. Dorotovič, R.J. Rutten Astronomical Society of the Pacific Conference Series, vol. 368, 2007, p. 611

M. Collados, R. López, E. Páez, E. Hernández, M. Reyes, A. Calcines, E. Ballesteros, J.J. Díaz, C. Denker, A. Lagg, R. Schlichenmaier, W. Schmidt, S.K. Solanki, K.G. Strassmeier, O. von der Lühe, R. Volkmer, GRIS: The GREGOR Infrared Spectrograph. Astronomische Nachrichten 333, 872 (2012). doi:10.1002/asna.201211738

M. Collados, F. Bettonvil, L. Cavaller, I. Ermolli, B. Gelly, A. Pérez, H. Socas-Navarro, D. Soltau, R. Volk- 
mer, EST Team, The European Solar Telescope. Memorie della Societa Astronomica Italiana 84, 379 (2013)

J. de la Cruz Rodríguez, H. Socas-Navarro, Are solar chromospheric fibrils tracing the magnetic field? Astron. Astrophys. 527, 8 (2011). doi:10.1051/0004-6361/201016018

J. de la Cruz Rodriguez, M. van Noort, H. Uitenbroek, Radiative diagnostics of the solar photosphere and chromosphere. (this issue) (2015)

J. de la Cruz Rodríguez, H. Socas-Navarro, M. Carlsson, J. Leenaarts, Non-local thermodynamic equilibrium inversions from a 3D magnetohydrodynamic chromospheric model. Astron. Astrophys. 543, 34 (2012). doi:10.1051/0004-6361/201218825

J. de la Cruz Rodríguez, L. Rouppe van der Voort, H. Socas-Navarro, M. van Noort, Physical properties of a sunspot chromosphere with umbral flashes. Astron. Astrophys. 556, 115 (2013). doi:10.1051/0004$6361 / 201321629$

B. De Pontieu, A.M. Title, J.R. Lemen, G.D. Kushner, D.J. Akin, B. Allard, T. Berger, P. Boerner, M. Cheung, C. Chou, J.F. Drake, D.W. Duncan, S. Freeland, G.F. Heyman, C. Hoffman, N.E. Hurlburt, R.W. Lindgren, D. Mathur, R. Rehse, D. Sabolish, R. Seguin, C.J. Schrijver, T.D. Tarbell, J.-P. Wülser, C.J. Wolfson, C. Yanari, J. Mudge, N. Nguyen-Phuc, R. Timmons, R. van Bezooijen, I. Weingrod, R. Brookner, G. Butcher, B. Dougherty, J. Eder, V. Knagenhjelm, S. Larsen, D. Mansir, L. Phan, P. Boyle, P.N. Cheimets, E.E. DeLuca, L. Golub, R. Gates, E. Hertz, S. McKillop, S. Park, T. Perry, W.A. Podgorski, K. Reeves, S. Saar, P. Testa, H. Tian, M. Weber, C. Dunn, S. Eccles, S.A. Jaeggli, C.C. Kankelborg, K. Mashburn, N. Pust, L. Springer, R. Carvalho, L. Kleint, J. Marmie, E. Mazmanian, T.M.D. Pereira, S. Sawyer, J. Strong, S.P. Worden, M. Carlsson, V.H. Hansteen, J. Leenaarts, M. Wiesmann, J. Aloise, K.-C. Chu, R.I. Bush, P.H. Scherrer, P. Brekke, J. Martinez-Sykora, B.W. Lites, S.W. McIntosh, H. Uitenbroek, T.J. Okamoto, M.A. Gummin, G. Auker, P. Jerram, P. Pool, N. Waltham, The Interface Region Imaging Spectrograph (IRIS). Solar Phys. 289, 2733-2779 (2014). doi:10.1007/s11207-014-0485-y

C. Debi Prasad, S. Gosain, Comparative Study of $\mathrm{LiNbO}_{3}$ and Servo Controlled Air Gap Fabry-Perot Etalons for Solar Application. Experimental Astronomy 13, 153-158 (2002). doi:10.1023/A:1025564731907

J.C. del Toro Iniesta, M. Collados, Optimum Modulation and Demodulation Matrices for Solar Polarimetry. Applied Optics 39, 1637-1642 (2000). doi:10.1364/AO.39.001637

C. Denker, H. Balthasar, A. Hofmann, N. Bello GonzÃjlez, R. Volkmer, 2010, The GREGOR Fabry-Perot interferometer: a new instrument for high-resolution solar observations. doi:10.1117/12.856830. http: //dx.doi.org/10.1117/12.856830

D.F. Elmore, T. Rimmele, R. Casini, S. Hegwer, J. Kuhn, H. Lin, J.P. McMullin, K. Reardon, W. Schmidt, A. Tritschler, F. Wöger, The Daniel K. Inouye Solar Telescope first light instruments and critical science plan, in Society of Photo-Optical Instrumentation Engineers (SPIE) Conference Series. Society of Photo-Optical Instrumentation Engineers (SPIE) Conference Series, vol. 9147, 2014, p. 7. doi:10.1117/12.2057038

A. Feller, F.A. Iglesias, K. Nagaraju, S.K. Solanki, S. Ihle, Fast Solar Polarimeter: Description and First Results, in Solar Polarization 7, ed. by K.N. Nagendra, J.O. Stenflo, Q. Qu, M. Samooprna Astronomical Society of the Pacific Conference Series, vol. 489, 2014, p. 271

A.M. Gandorfer, H.P.P.P. Steiner, F. Aebersold, U. Egger, A. Feller, D. Gisler, S. Hagenbuch, J.O. Stenflo, Solar polarimetry in the near UV with the Zurich Imaging Polarimeter ZIMPOL II. Astron. Astrophys. 422, 703-708 (2004). doi:10.1051/0004-6361:20040254

A. Gandorfer, B. Grauf, P. Barthol, T.L. Riethmüller, S.K. Solanki, B. Chares, W. Deutsch, S. Ebert, A. Feller, D. Germerott, K. Heerlein, J. Heinrichs, D. Hirche, J. Hirzberger, M. Kolleck, R. Meller, R. Müller, R. Schäfer, G. Tomasch, M. Knölker, V. Martínez Pillet, J.A. Bonet, W. Schmidt, T. Berkefeld, B. Feger, F. Heidecke, D. Soltau, A. Tischenberg, A. Fischer, A. Title, H. Anwand, E. Schmidt, The Filter Imager SuFI and the Image Stabilization and Light Distribution System ISLiD of the Sunrise Balloon-Borne Observatory: Instrument Description. Solar Phys. 268, 35-55 (2011). doi:10.1007/s11207-010-9636-y

P.R. Goode, R. Coulter, N. Gorceix, V. Yurchyshyn, W. Cao, The NST: First results and some lessons for ATST and EST. Astronomische Nachrichten 331, 620 (2010). doi:10.1002/asna.201011387

S. Gosain, P. Venkatakrishnan1, K. Venugopalan, Design and Status of Solar Vector Magnetograph (SVM-I) at Udaipur Solar Observatory. Journal of Astrophysics and Astronomy 27, 285-292 (2006). doi:10.1007/BF02702531

S. Gosain, A.A. Pevtsov, G.V. Rudenko, S.A. Anfinogentov, First Synoptic Maps of Photospheric Vector Magnetic Field from SOLIS/VSM: Non-radial Magnetic Fields and Hemispheric Pattern of Helicity. Astrophys. J. 772, 52 (2013). doi:10.1088/0004-637X/772/1/52

B.V. Gudiksen, M. Carlsson, V.H. Hansteen, W. Hayek, J. Leenaarts, J. Martínez-Sykora, The stellar atmosphere simulation code Bifrost. Code description and validation. Astron. Astrophys. 531, 154 (2011). doi:10.1051/0004-6361/201116520

N. Hagen, M.W. Kudenov, Review of snapshot spectral imaging technologies. Optical Engineering 52(9), 
090901-090901 (2013)

G.E. Hale, On the Probable Existence of a Magnetic Field in Sun-Spots. ApJ 28, 315 (1908). doi:10.1086/141602

J.W. Harvey, F. Hill, R.P. Hubbard, J.R. Kennedy, J.W. Leibacher, J.A. Pintar, P.A. Gilman, R.W. Noyes, A.M. Title, J. Toomre, R.K. Ulrich, A. Bhatnagar, J.A. Kennewell, W. Marquette, J. Patron, O. Saa, E. Yasukawa, The Global Oscillation Network Group (GONG) Project. Science 272, 1284-1286 (1996). doi:10.1126/science.272.5266.1284

F. Hill, M.J. Thompson, M. Roth, Workshop Report: A New Synoptic Solar Observing Network. Space Weather 11, 392-393 (2013). doi:10.1002/swe.20068

C.L. Hyder, Winking Filaments and Prominence and Coronal Magnetic Fields. Zeitschrift f $\tilde{A} \frac{1}{4} \mathrm{r}$ Astrophysik 63, 78 (1966)

H. Ito, S. Tsuneta, D. Shiota, M. Tokumaru, K. Fujiki, Is the Polar Region Different from the Quiet Region of the Sun? ApJ 719, 131-142 (2010). doi:10.1088/0004-637X/719/1/131

S.A. Jaeggli, H. Lin, D.L. Mickey, J.R. Kuhn, S.L. Hegwer, T.R. Rimmele, M.J. Penn, FIRS: a new instrument for photospheric and chromospheric studies at the DST. Memorie della Societa Astronomica Italiana 81, 763 (2010)

P. Judge, S. White, R. Casini, Coronal measurements. (this issue) (2015)

P.G. Judge, D.F. Elmore, B.W. Lites, C.U. Keller, T. Rimmele, Evaluation of Seeing-Induced Cross Talk in Tip-Tilt-Corrected Solar Polarimetry. Applied Optics 43, 3817-3828 (2004). doi:10.1364/AO.43.003817

C.U. Keller, Instrumentation for astrophysical spectropolarimetry, in Astrophysical Spectropolarimetry, ed. by J. Trujillo-Bueno, F. Moreno-Insertis, F. Sánchez, 2002, pp. 303-354

C.U. Keller, Solis Team, The SOLIS Vector-Spectromagnetograph (VSM), in Advanced Solar Polarimetry - Theory, Observation, and Instrumentation, ed. by M. Sigwarth Astronomical Society of the Pacific Conference Series, vol. 236, 2001, p. 16

C.U. Keller, O. von der Luehe, Solar speckle polarimetry. Astron. Astrophys. 261, 321-328 (1992)

L. Kleint, Spectropolarimetry of C-class Flare Footpoints. Astrophys. J. 748, 138 (2012). doi:10.1088/0004$637 \mathrm{X} / 748 / 2 / 138$

L. Kleint, A. Gandorfer, Prospects of solar magnetometry - from ground and in space. ArXiv e-prints (2015). this issue. doi:10.1007/s11214-015-0208-1

R. Komm, S. Gosain, Current and Kinetic Helicity of Long-lived Activity Complexes. Astrophys. J. 798, 20 (2015). doi:10.1088/0004-637X/798/1/20

R. Komm, S. Gosain, A.A. Pevtsov, Hemispheric Distribution of Subsurface Kinetic Helicity and Its Variation with Magnetic Activity. Solar Phys. 289, 2399-2418 (2014). doi:10.1007/s11207-014-0477-y

T. Kosugi, K. Matsuzaki, T. Sakao, T. Shimizu, Y. Sone, S. Tachikawa, T. Hashimoto, K. Minesugi, A. Ohnishi, T. Yamada, S. Tsuneta, H. Hara, K. Ichimoto, Y. Suematsu, M. Shimojo, T. Watanabe, S. Shimada, J.M. Davis, L.D. Hill, J.K. Owens, A.M. Title, J.L. Culhane, L.K. Harra, G.A. Doschek, L. Golub, The Hinode (Solar-B) Mission: An Overview. Sol. Phys. 243, 3-17 (2007). doi:10.1007/s11207007-9014-6

N. Krishnappa, A. Feller, Precision in ground-based solar polarimetry: simulating the role of adaptive optics. Applied Optics 51, 7953 (2012). doi:10.1364/AO.51.007953

M.R. Kundu, Measurement of solar magnetic fields from radio observations. Società Astronomica Italiana, Memorie (ISSN 0037-8720) 61, 431-455 (1990)

A. Lagg, R. Ishikawa, L. Merenda, T. Wiegelmann, S. Tsuneta, S.K. Solanki, Internetwork Horizontal Magnetic Fields in the Quiet Sun Chromosphere: Results from a Joint Hinode/VTT Study, in The Second Hinode Science Meeting: Beyond Discovery-Toward Understanding, ed. by B. Lites, M. Cheung, T. Magara, J. Mariska, K. Reeves Astronomical Society of the Pacific Conference Series, vol. 415, 2009, p. 327

A. Lagg, S.K. Solanki, M. van Noort, S. Danilovic, Vigorous convection in a sunspot granular light bridge. Astron. Astrophys. 568, 60 (2014). doi:10.1051/0004-6361/201424071

E. Landi Degl'Innocenti, M. Landolfi (eds.), Polarization in Spectral Lines, in Astrophysics and Space Science Library, Astrophysics and Space Science Library, vol. 3072004

J. Leenaarts, M. Carlsson, L. Rouppe van der Voort, The Formation of the H $\alpha$ Line in the Solar Chromosphere. Astrophys. J. 749, 136 (2012). doi:10.1088/0004-637X/749/2/136

J. Leenaarts, M. Carlsson, V. Hansteen, L. Rouppe van der Voort, Three-Dimensional Non-LTE Radiative Transfer Computation of the CA 8542 Infrared Line From a Radiation-MHD Simulation. ApJL 694, 128-131 (2009). doi:10.1088/0004-637X/694/2/L128

K.D. Leka, K.E. Rangarajan, Effects of 'Seeing' on Vector Magnetograph Measurements. Solar Phys. 203, 239-254 (2001). doi:10.1023/A:1013373424510

B.W. Lites, Rotating waveplates as polarization modulators for Stokes polarimetry of the sun - Evaluation of 
seeing-induced crosstalk errors. Applied Optics 26, 3838-3845 (1987). doi:10.1364/AO.26.003838

B.W. Lites, Performance Characteristics of the Advanced Stokes Polarimeter. Solar Phys. 163, 223-230 (1996). doi:10.1007/BF00147996

B.W. Lites, V. Martinez Pillet, A. Skumanich, A quantitative comparison of vector magnetic field measurement and analysis techniques. Solar Phys. 155, 1-27 (1994). doi:10.1007/BF00670727

B.W. Lites, K.D. Leka, A. Skumanich, V. Martinez Pillet, T. Shimizu, Small-Scale Horizontal Magnetic Fields in the Solar Photosphere. ApJ 460, 1019 (1996). doi:10.1086/177028

B.W. Lites, M. Kubo, H. Socas-Navarro, T. Berger, Z. Frank, R. Shine, T. Tarbell, A. Title, K. Ichimoto, Y. Katsukawa, S. Tsuneta, Y. Suematsu, T. Shimizu, S. Nagata, The Horizontal Magnetic Flux of the Quiet-Sun Internetwork as Observed with the Hinode Spectro-Polarimeter. ApJ 672, 1237-1253 (2008). doi:10.1086/522922

B.W. Lites, D.L. Akin, G. Card, T. Cruz, D.W. Duncan, C.G. Edwards, D.F. Elmore, C. Hoffmann, Y. Katsukawa, N. Katz, M. Kubo, K. Ichimoto, T. Shimizu, R.A. Shine, K.V. Streander, A. Suematsu, T.D. Tarbell, A.M. Title, S. Tsuneta, The Hinode Spectro-Polarimeter. Sol. Phys. 283, 579-599 (2013). doi:10.1007/s11207-012-0206-3

Y. Liu, J.T. Hoeksema, X. Sun, Test of the Hemispheric Rule of Magnetic Helicity in the Sun Using the Helioseismic and Magnetic Imager (HMI) Data. ApJL 783, 1 (2014). doi:10.1088/2041-8205/783/1/L1

M.G. Lofdahl, G.B. Scharmer, Application of phase-diversity to solar images, in Image Reconstruction and Restoration, ed. by T.J. Schulz, D.L. Snyder Society of Photo-Optical Instrumentation Engineers (SPIE) Conference Series, vol. 2302, 1994, pp. 254-267

M.G. Löfdahl, G.B. Scharmer, Wavefront sensing and image restoration from focused and defocused solar images. Astronomy and Astrophysics Suppl. 107, 243-264 (1994)

S.F. Martin, H.E. Ramsey, G.A. Carroll, D.C. Martin, Multi-slit spectrograph and H alpha Doppler system. Solar Phys. 37, 343-350 (1974). doi:10.1007/BF00152493

M.J. Martínez González, R. Manso Sainz, A. Asensio Ramos, E. Hijano, Dead Calm Areas in the Very Quiet Sun. ApJ 755, 175 (2012). doi:10.1088/0004-637X/755/2/175

V. Martínez Pillet, J.C. Del Toro Iniesta, A. Álvarez-Herrero, V. Domingo, J.A. Bonet, L. González Fernández, A. López Jiménez, C. Pastor, J.L. Gasent Blesa, P. Mellado, J. Piqueras, B. Aparicio, M. Balaguer, E. Ballesteros, T. Belenguer, L.R. Bellot Rubio, T. Berkefeld, M. Collados, W. Deutsch, A. Feller, F. Girela, B. Grauf, R.L. Heredero, M. Herranz, J.M. Jerónimo, H. Laguna, R. Meller, M. Menéndez, R. Morales, D. Orozco Suárez, G. Ramos, M. Reina, J.L. Ramos, P. Rodríguez, A. Sánchez, N. UribePatarroyo, P. Barthol, A. Gandorfer, M. Knoelker, W. Schmidt, S.K. Solanki, S. Vargas Domínguez, The Imaging Magnetograph eXperiment (IMaX) for the Sunrise Balloon-Borne Solar Observatory. Solar Phys. 268, 57-102 (2011). doi:10.1007/s11207-010-9644-y

S.K. Mathew, A. Bhatnagar, C.D. Prasad, A. Ambastha, Fabry-Perot filter based solar video magnetograph. Astronomy and Astrophysics Supplement 133, 285-292 (1998). doi:10.1051/aas:1998312

L. Merenda, J. Trujillo Bueno, E. Landi Degl'Innocenti, M. Collados, Determination of the Magnetic Field Vector via the Hanle and Zeeman Effects in the He I 110830 Multiplet: Evidence for Nearly Vertical Magnetic Fields in a Polar Crown Prominence. ApJ 642, 554-561 (2006). doi:10.1086/501038

T.R. Metcalf, K.D. Leka, G. Barnes, B.W. Lites, M.K. Georgoulis, A.A. Pevtsov, K.S. Balasubramaniam, G.A. Gary, J. Jing, J. Li, Y. Liu, H.N. Wang, V. Abramenko, V. Yurchyshyn, Y.-J. Moon, An Overview of Existing Algorithms for Resolving the $180^{\circ}$ Ambiguity in Vector Magnetic Fields: Quantitative Tests with Synthetic Data. Solar Phys. 237, 267-296 (2006). doi:10.1007/s11207-006-0170-x

D.L. Mickey, R.C. Canfield, B.J. Labonte, K.D. Leka, M.F. Waterson, H.M. Weber, The Imaging Vector Magnetograph at Haleakala. Solar Phys. 168, 229-250 (1996). doi:10.1007/BF00148052

D. Neidig, P. Wiborg, M. Confer, B. Haas, R. Dunn, K.S. Balasubramaniam, C. Gullixson, D. Craig, M. Kaufman, W. Hull, R. McGraw, T. Henry, R. Rentschler, C. Keller, H. Jones, R. Coulter, S. Gregory, R. Schimming, B. Smaga, The USAF Improved Solar Observing Optical Network (ISOON) and its Impact on Solar Synoptic Data Bases, in Synoptic Solar Physics, ed. by K.S. Balasubramaniam, J. Harvey, D. Rabin Astronomical Society of the Pacific Conference Series, vol. 140, 1998, p. 519

D. Orozco Suárez, A. Asensio Ramos, J. Trujillo Bueno, The magnetic field configuration of a solar prominence inferred from spectropolarimetric observations in the He i $10830 \AA$ triplet. Astron. Astrophys. 566, 46 (2014). doi:10.1051/0004-6361/201322903

D. Orozco Suárez, A. Asensio Ramos, J. Trujillo Bueno, Height Variation of the Vector Magnetic Field in Solar Spicules. ApJL 803, 18 (2015). doi:10.1088/2041-8205/803/2/L18

G. Petrie, S. Ettinger, Polar Field Reversals and Active Region Decay. Space Science Reviews (2015). this issue. doi:10.1007/s11214-015-0189-0

A.A. Pevtsov, R.C. Canfield, T.R. Metcalf, Patterns of helicity in solar active regions. ApJL 425, 117-119 (1994). doi:10.1086/187324

A. Pietarila, H. Socas-Navarro, T. Bogdan, Spectropolarimetric Inversions of the Ca II 8498 and 8542 A Lines 
in the Quiet Sun. Astrophys. J. 670, 885-902 (2007). doi:10.1086/521271

J. Pietarila Graham, S. Danilovic, M. Schüssler, Turbulent Magnetic Fields in the Quiet Sun: Implications of Hinode Observations and Small-Scale Dynamo Simulations. ApJ 693, 1728-1735 (2009). doi:10.1088/0004-637X/693/2/1728

H.P. Povel, C.U. Keller, I.-A. Yadigaroglu, Two-dimensional polarimeter with a charge-coupled-device image sensor and a piezoelastic modulator. Applied Optics 33, 4254-4260 (1994). doi:10.1364/AO.33.004254

K.G. Puschmann, C. Denker, F. Kneer, N. Al Erdogan, H. Balthasar, S.M. Bauer, C. Beck, N. Bello González, M. Collados, T. Hahn, J. Hirzberger, A. Hofmann, R.E. Louis, H. Nicklas, O. Okunev, V. Martínez Pillet, E. Popow, T. Seelemann, R. Volkmer, A.D. Wittmann, M. Woche, The GREGOR Fabry-Pérot Interferometer. Astronomische Nachrichten 333, 880 (2012). doi:10.1002/asna.201211734

R. Ramelli, S. Balemi, M. Bianda, I. Defilippis, L. Gamma, S. Hagenbuch, M. Rogantini, P. Steiner, J.O. Stenflo, ZIMPOL-3: a powerful solar polarimeter, in Society of Photo-Optical Instrumentation Engineers (SPIE) Conference Series. Society of Photo-Optical Instrumentation Engineers (SPIE) Conference Series, vol. 7735 , 2010, p. 1. doi:10.1117/12.857120

M. Rempel, Numerical Simulations of Quiet Sun Magnetism: On the Contribution from a Small-scale Dynamo. Astrophys. J. 789, 132 (2014). doi:10.1088/0004-637X/789/2/132

M. Rempel, G. Scharmer, Sunspots. (this issue) (2015)

D. Ren, L. Jolissaint, X. Zhang, J. Dou, R. Chen, G. Zhao, Y. Zhu, Solar Ground-Layer Adaptive Optics. Publ. Astron. Soc. Pacific 127, 469-478 (2015). doi:10.1086/681672

T.R. Rimmele, Solar adaptive optics, in Adaptive Optical Systems Technology, ed. by P.L. Wizinowich Society of Photo-Optical Instrumentation Engineers (SPIE) Conference Series, vol. 4007, 2000, pp. 218-231

T.R. Rimmele, ATST Team, The unique scientific capabilities of the Advanced Technology Solar Telescope. Advances in Space Research 42, 78-85 (2008). doi:10.1016/j.asr.2008.02.024

T.R. Rimmele, J. Marino, Solar Adaptive Optics. Living Reviews in Solar Physics 8, 2 (2011). doi:10.12942/lrsp-2011-2

T.R. Rimmele, F. Woeger, J. Marino, K. Richards, S. Hegwer, T. Berkefeld, D. Soltau, D. Schmidt, T. Waldmann, Solar multiconjugate adaptive optics at the Dunn Solar Telescope, in Society of Photo-Optical Instrumentation Engineers (SPIE) Conference Series. Society of Photo-Optical Instrumentation Engineers (SPIE) Conference Series, vol. 7736, 2010, p. 31. doi:10.1117/12.857485

M. Rodenhuis, F. Snik, G. van Harten, J. Hoeijmakers, C.U. Keller, Five-dimensional optical instrumentation: combining polarimetry with time-resolved integral-field spectroscopy, in Polarization: Measurement, Analysis, and Remote Sensing XI, ed. by D.B. Chenault, D.H. Goldstein Society of PhotoOptical Instrumentation Engineers (SPIE) Conference Series, vol. 9099, 2014. doi:10.1117/12.2053241. http://dx.doi.org/10.1117/12.2053241

C. Sasso, A. Lagg, S.K. Solanki, Milne-Eddington inversions of the He I $10830 \AA$ A Stokes profiles: influence of the Paschen-Back effect. A\&A 456, 367-371 (2006). doi:10.1051/0004-6361:20065257

T.A. Schad, Spectropolarimetry of fine magnetized structures in the upper solar atmosphere, $\mathrm{PhD}$ thesis, The University of Arizona, 2013

T.A. Schad, M.J. Penn, H. Lin, He I Vector Magnetometry of Field-aligned Superpenumbral Fibrils. Astrophys. J. 768, 111 (2013). doi:10.1088/0004-637X/768/2/111

G.B. Scharmer, Comments on the optimization of high resolution Fabry-Pérot filtergraphs. Astron. Astrophys. 447, 1111-1120 (2006). doi:10.1051/0004-6361:20052981

G.B. Scharmer, M. Shand, M.G. Lofdahl, P.M. Dettori, W. Wei, Workstation-based solar/stellar adaptive optics system, in Adaptive Optical Systems Technology, ed. by P.L. Wizinowich Society of Photo-Optical Instrumentation Engineers (SPIE) Conference Series, vol. 4007, 2000, pp. 239-250

G.B. Scharmer, K. Bjelksjo, T.K. Korhonen, B. Lindberg, B. Petterson, The 1-meter Swedish solar telescope, in Innovative Telescopes and Instrumentation for Solar Astrophysics, ed. by S.L. Keil, S.V. Avakyan Society of Photo-Optical Instrumentation Engineers (SPIE) Conference Series, vol. 4853, 2003, pp. 341350

G.B. Scharmer, G. Narayan, T. Hillberg, J. de la Cruz Rodríguez, M.G. Löfdahl, D. Kiselman, P. Sütterlin, M. van Noort, A. Lagg, CRISP Spectropolarimetric Imaging of Penumbral Fine Structure. ApJL 689, 69-72 (2008). doi:10.1086/595744

G.B. Scharmer, J. de la Cruz Rodriguez, P. Sütterlin, V.M.J. Henriques, Opposite polarity field with convective downflow and its relation to magnetic spines in a sunspot penumbra. Astron. Astrophys. 553, 63 (2013). doi:10.1051/0004-6361/201220899

P.H. Scherrer, R.S. Bogart, R.I. Bush, J.T. Hoeksema, A.G. Kosovichev, J. Schou, W. Rosenberg, L. Springer, T.D. Tarbell, A. Title, C.J. Wolfson, I. Zayer, MDI Engineering Team, The Solar Oscillations Investigation - Michelson Doppler Imager. Sol. Phys. 162, 129-188 (1995)

P.H. Scherrer, J. Schou, R.I. Bush, A.G. Kosovichev, R.S. Bogart, J.T. Hoeksema, Y. Liu, T.L. Duvall, J. Zhao, A.M. Title, C.J. Schrijver, T.D. Tarbell, S. Tomczyk, The Helioseismic and Magnetic Imager 
(HMI) Investigation for the Solar Dynamics Observatory (SDO). Solar Phys. 275, 207-227 (2012). doi:10.1007/s11207-011-9834-2

D. Schmidt, T. Berkefeld, F. Heidecke, A. Fischer, O. von der Lühe, D. Soltau, GREGOR MCAO looking at the Sun, in Society of Photo-Optical Instrumentation Engineers (SPIE) Conference Series. Society of Photo-Optical Instrumentation Engineers (SPIE) Conference Series, vol. 9148, 2014, p. 1. doi:10.1117/12.2055154

W. Schmidt, O. von der Lühe, R. Volkmer, C. Denker, S.K. Solanki, H. Balthasar, N. Bello Gonzalez, T. Berkefeld, M. Collados, A. Fischer, C. Halbgewachs, F. Heidecke, A. Hofmann, F. Kneer, A. Lagg, H. Nicklas, E. Popow, K.G. Puschmann, D. Schmidt, M. Sigwarth, M. Sobotka, D. Soltau, J. Staude, K.G. Strassmeier, T.A. Waldmann, The 1.5 meter solar telescope GREGOR. Astronomische Nachrichten 333, 796 (2012). doi:10.1002/asna.201211725

J. Schou, P.H. Scherrer, R.I. Bush, R. Wachter, S. Couvidat, M.C. Rabello-Soares, R.S. Bogart, J.T. Hoeksema, Y. Liu, T.L. Duvall, D.J. Akin, B.A. Allard, J.W. Miles, R. Rairden, R.A. Shine, T.D. Tarbell, A.M. Title, C.J. Wolfson, D.F. Elmore, A.A. Norton, S. Tomczyk, Design and Ground Calibration of the Helioseismic and Magnetic Imager (HMI) Instrument on the Solar Dynamics Observatory (SDO). Solar Phys. 275, 229-259 (2012). doi:10.1007/s11207-011-9842-2

D. Shiota, S. Tsuneta, M. Shimojo, N. Sako, D. Orozco Suárez, R. Ishikawa, Polar Field Reversal Observations with Hinode. ApJ 753, 157 (2012). doi:10.1088/0004-637X/753/2/157

F. Snik, J. Craven-Jones, M. Escuti, S. Fineschi, D. Harrington, A.D. Martino, D. Mawet, J. Riedi, J.S. Tyo, An overview of polarimetric sensing techniques and technology with applications to different research fields, in Polarization: Measurement, Analysis, and Remote Sensing XI, ed. by D.B. Chenault, D.H. Goldstein Society of Photo-Optical Instrumentation Engineers (SPIE) Conference Series, vol. 9099, 2014. doi:10.1117/12.2053245. http://dx.doi.org/10.1117/12.2053245

H. Socas-Navarro, J. Trujillo Bueno, E. Landi Degl'Innocenti, Signatures of incomplete paschen-back splitting in the polarization profiles of the he i $\lambda 10830$ multiplet. ApJ 612, 1175-1180 (2004)

H. Socas-Navarro, J. Trujillo Bueno, E. Landi Degl'Innocenti, Polynomial Approximants for the Calculation of Polarization Profiles in the He I 10830 A Multiplet. ApJS 160, 312-317 (2005). doi:10.1086/431934

H. Socas-Navarro, J. Trujillo Bueno, B. Ruiz Cobo, Non-LTE Inversion of Stokes Profiles Induced by the Zeeman Effect. Astrophys. J. 530, 977-993 (2000). doi:10.1086/308414

H. Socas-Navarro, D. Elmore, A. Pietarila, A. Darnell, B.W. Lites, S. Tomczyk, S. Hegwer, Spinor: Visible and Infrared Spectro-Polarimetry at the National Solar Observatory. Solar Phys. 235, 55-73 (2006). doi:10.1007/s11207-006-0020-x

S.K. Solanki, N.A. Krivova, J.D. Haigh, Solar Irradiance Variability and Climate. Annual Review of Astronomy and Astrophysics 51, 311-351 (2013). doi:10.1146/annurev-astro-082812-141007

L. Staveland, Determination of the Spread Function for Solar Stray Light. Solar Phys. 12, 328-331 (1970). doi:10.1007/BF00227125

J. Stenflo (ed.), Solar Magnetic Fields: Polarized Radiation Diagnostics, in Astrophysics and Space Science Library, Astrophysics and Space Science Library, vol. 1891994

J.O. Stenflo, Solar magnetic fields as revealed by Stokes polarimetry. The Astronomy and Astrophysics Review 21, 66 (2013). doi:10.1007/s00159-013-0066-3

J.O. Stenflo, History of Solar Magnetic Fields Since George Ellery Hale. Space Science Reviews (2015). this issue. doi:10.1007/s11214-015-0198-z

J.O. Stenflo, A.G. Kosovichev, Bipolar Magnetic Regions on the Sun: Global Analysis of the SOHO/MDI Data Set. ApJ 745, 129 (2012). doi:10.1088/0004-637X/745/2/129

T. Tadesse, T. Wiegelmann, S. Gosain, P. MacNeice, A.A. Pevtsov, First use of synoptic vector magnetograms for global nonlinear, force-free coronal magnetic field models. Astron. Astrophys. 562, 105 (2014a). doi:10.1051/0004-6361/201322418

T. Tadesse, A.A. Pevtsov, T. Wiegelmann, P.J. MacNeice, S. Gosain, Global Solar Free Magnetic Energy and Electric Current Density Distribution of Carrington Rotation 2124. Solar Phys. 289, 4031-4045 (2014b). doi:10.1007/s11207-014-0581-z

S.K. Tiwari, M. van Noort, A. Lagg, S.K. Solanki, Structure of sunspot penumbral filaments: a remarkable uniformity of properties. Astron. Astrophys. 557, 25 (2013). doi:10.1051/0004-6361/201321391

S. Trippe, [Review] Polarization and Polarimetry. Journal of Korean Astronomical Society 47, 15-39 (2014). doi:10.5303/JKAS.2014.47.1.15

A. Tritschler, T.R. Rimmele, S. Berukoff, R. Casini, S.C. Craig, D.F. Elmore, R.P. Hubbard, J.R. Kuhn, H. Lin, J.P. McMullin, K.P. Reardon, W. Schmidt, M. Warner, F. Woger, DKIST: Observing the Sun at High Resolution, in 18th Cambridge Workshop on Cool Stars, Stellar Systems, and the Sun, ed. by G.T. van Belle, H.C. Harris Cambridge Workshop on Cool Stars, Stellar Systems, and the Sun, vol. 18, 2015, pp. 933-944

J. Trujillo Bueno, Polarized Radiation Observables for Probing the Magnetism of the Outer Solar Atmo- 
sphere, in Solar Polarization 7, ed. by K.N. Nagendra, J.O. Stenflo, Q. Qu, M. Samooprna Astronomical Society of the Pacific Conference Series, vol. 489, 2014, p. 137

J. Trujillo Bueno, E. Landi degl'Innocenti, UV Polarized Radiation Observables for Probing the Upper Chromosphere. (this issue) (2015)

J. Trujillo Bueno, E. Landi Degl'Innocenti, M. Collados, L. Merenda, R. Manso Sainz, Selective absorption processes as the origin of puzzling spectral line polarization from the Sun. Nature 415, 403-406 (2002)

S. Tsuneta, K. Ichimoto, Y. Katsukawa, S. Nagata, M. Otsubo, T. Shimizu, Y. Suematsu, M. Nakagiri, M. Noguchi, T. Tarbell, A. Title, R. Shine, W. Rosenberg, C. Hoffmann, B. Jurcevich, G. Kushner, M. Levay, B. Lites, D. Elmore, T. Matsushita, N. Kawaguchi, H. Saito, I. Mikami, L.D. Hill, J.K. Owens, The Solar Optical Telescope for the Hinode Mission: An Overview. Sol. Phys. 249, 167-196 (2008). doi:10.1007/s11207-008-9174-z

P.J. Turon, P.J. Léna, High resolution solar images at 10 microns: Sunspot details and photometry. Solar Phys. 14, 112-124 (1970). doi:10.1007/BF00240164

M. van Noort, Spatially coupled inversion of spectro-polarimetric image data. I. Method and first results. Astron. Astrophys. 548, 5 (2012). doi:10.1051/0004-6361/201220220

M. van Noort, L. Rouppe van der Voort, M.G. Löfdahl, Solar Image Restoration By Use Of Multi-frame Blind De-convolution With Multiple Objects And Phase Diversity. Solar Phys. 228, 191-215 (2005). doi:10.1007/s11207-005-5782-z

A. Vögler, M. Schüssler, A solar surface dynamo. A\&A 465, 43-46 (2007). doi:10.1051/00046361:20077253

O. von der Luehe, Speckle imaging of solar small scale structure. I - Methods. Astron. Astrophys. 268, 374390 (1993)

S. Wedemeyer, T. Bastian, R. Brajsa, M. Barta, H. Hudson, G. Fleishman, M. Loukitcheva, B. Fleck, E.P. Kontar, B. De Pontieu, S.K. Tiwari, Y. Kato, R. Soler, P. Yagoubov, J.H. Black, P. Antolin, E. Scullion, S. Gun'ar, N. Labrosse, A.O. Benz, H.-G. Ludwig, P. Hauschildt, J.G. Doyle, V.M. Nakariakov, S.K. Solanki, S.M. White, T. Ayres, P. Heinzel, M. Karlicky, T. Van Doorsselaere, D. Gary, C.E. Alissandrakis, A. Nindos, L. Rouppe van der Voort, M. Shimojo, T. Zaqarashvili, E. Perez, Solar science with the Atacama Large Millimeter/submillimeter Array - A revolutionizing new view of our Sun. ArXiv e-prints (2015)

T. Wiegelmann, G.J.D. Petrie, P. Riley, Coronal Magnetic Field Models. Space Science Reviews (2015). this issue. doi: $10.1007 / \mathrm{s} 11214-015-0178-3$

T. Wiegelmann, J.K. Thalmann, C.J. Schrijver, M.L. Derosa, T.R. Metcalf, Can We Improve the Preprocessing of Photospheric Vector Magnetograms by the Inclusion of Chromospheric Observations? Sol. Phys. 247, 249-267 (2008). doi:10.1007/s11207-008-9130-y 